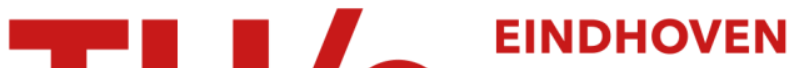 UNIVERSITY OF TECHNOLOGY
}

\section{Sparse iterative learning control with application to a wafer stage}

Citation for published version (APA):

Oomen, T. A. E., \& Rojas, C. R. (2017). Sparse iterative learning control with application to a wafer stage: achieving performance, resource efficiency, and task flexibility. Mechatronics, 47, 134-147.

https://doi.org/10.1016/j.mechatronics.2017.09.004

\section{Document license:}

TAVERNE

DOI:

10.1016/j.mechatronics.2017.09.004

Document status and date:

Published: 01/11/2017

\section{Document Version:}

Publisher's PDF, also known as Version of Record (includes final page, issue and volume numbers)

\section{Please check the document version of this publication:}

- A submitted manuscript is the version of the article upon submission and before peer-review. There can be important differences between the submitted version and the official published version of record. People interested in the research are advised to contact the author for the final version of the publication, or visit the $\mathrm{DOI}$ to the publisher's website.

- The final author version and the galley proof are versions of the publication after peer review.

- The final published version features the final layout of the paper including the volume, issue and page numbers.

Link to publication

\section{General rights}

Copyright and moral rights for the publications made accessible in the public portal are retained by the authors and/or other copyright owners and it is a condition of accessing publications that users recognise and abide by the legal requirements associated with these rights.

- Users may download and print one copy of any publication from the public portal for the purpose of private study or research.

- You may not further distribute the material or use it for any profit-making activity or commercial gain

- You may freely distribute the URL identifying the publication in the public portal.

If the publication is distributed under the terms of Article 25fa of the Dutch Copyright Act, indicated by the "Taverne" license above, please follow below link for the End User Agreement:

www.tue.nl/taverne

Take down policy

If you believe that this document breaches copyright please contact us at:

openaccess@tue.nl

providing details and we will investigate your claim. 


\title{
Sparse iterative learning control with application to a wafer stage: Achieving performance, resource efficiency, and task flexibility ${ }^{\text {is }}$
}

\author{
Tom Oomen ${ }^{\mathrm{a}, *}$, Cristian R. Rojas ${ }^{\mathrm{b}}$ \\ ${ }^{a}$ Eindhoven University of Technology, Faculty of Mechanical Engineering, Control Systems Technology group, PO Box 513, Eindhoven 5600MB, The \\ Netherlands \\ ${ }^{\mathrm{b}}$ Automatic Control Lab, Electrical Engineering, KTH - Royal Institute of Technology, Stockholm S-100 44, Sweden
}

\section{A R T I C L E I N F O}

\section{Article history:}

Received 5 June 2017

Revised 7 August 2017

Accepted 13 September 2017

Available online 6 October 2017

\section{Keywords:}

Iterative learning control

Motion control

Feedforward

Sparse optimization

Resource-efficient control

\begin{abstract}
A B S T R A C T
Trial-varying disturbances are a key concern in Iterative Learning Control (ILC) and may lead to inefficient and expensive implementations and severe performance deterioration. The aim of this paper is to develop a general framework for optimization-based ILC that allows for enforcing additional structure, including sparsity. The proposed method enforces sparsity in a generalized setting through convex relaxations using $\ell_{1}$ norms. The proposed ILC framework is applied to the optimization of sampling sequences for resource efficient implementation, trial-varying disturbance attenuation, and basis function selection. The framework has a large potential in control applications such as mechatronics, as is confirmed through an application on a wafer stage.
\end{abstract}

(ㄷ) 2017 Elsevier Ltd. All rights reserved.

\section{Introduction}

Iterative Learning Control (ILC) enables significant performance improvements for batch-to-batch control applications, by generating a command signal that compensates for repetitive disturbances through learning from previous iterations, also called batches or trials. Theoretical and implementation aspects, including convergence, causality, and robustness, have been addressed in, e.g., $[1,12,41,44,45]$. Furthermore, successful applications have been reported in, e.g., robotics [53], mechatronics [9], manufacturing [27], building control [43], nuclear fusion [19], and rehabilitation [20]. However, several disadvantages of present ILC frameworks that limit further applications include (i) high implementation cost due to highly unstructured command signals, which are expensive to implement; (ii) amplification of trial-varying disturbances, including measurement noise; (iii) inflexibility to changing reference trajectories. The aim of the present paper is to develop an ILC design framework that enforces sparsity, which enables addressing these aspects (i)-(iii).

Regarding (i) ILC typically generates signals that require a large number of command signal updates thus leading to an expensive implementation. ILC directly learns from measured signals that are

\footnotetext{
This paper was recommended for publication by Associate Editor Dr Peter Hehenberger.

* Corresponding author.

E-mail address: t.a.e.oomen@tue.nl (T. Oomen).
}

contaminated by trial-varying disturbances such as measurement noise. These trial-varying disturbances are often modeled as a realization of a stochastic process [32]. As a result, the ILC command signals have infinite support. In sharp contrast, command signals that are obtained through traditional feedforward designs, including [31], have finite support and are highly sparse. Command signals with a high number of non-zero elements, or another appropriate structural constraint, may lead to a prohibitively expensive implementation, e.g., in wireless sensor networks, wireless control applications, or embedded platforms with shared resources [22]. Note that this is a different aspect than the actual computation of the command signal itself, which can be done in between subsequent tasks, see [60] for results in this direction.

Regarding (ii), ILC typically amplifies trial-varying disturbances. In fact, typical ILC approaches amplify these disturbances by a factor of two, as is shown in the present paper. Approaches to attenuate trial-varying disturbances include norm-optimal ILC with appropriate input weighting [12], higher-order ILC for addressing disturbances with trial-domain dynamics [24], and stochastic approximation-based ILC [14]. Also, a wavelet filtering-based approach is presented in [33], where a certain noise attenuation is achieved by setting certain wavelet coefficients to zero. In the present paper, a different approach is pursued to attenuate disturbances, where also wavelets immediately fit into the formulation, yet the sparsity can be enforced in an optimal way.

Regarding (iii), changing reference signals typically lead to performance degradation of ILC algorithms [5], since these essentially 
constitute trial-varying disturbances. This is in sharp contrast to traditional feedforward designs [31] and is widely recognized in ILC designs. A basis task approach is proposed in [26], where the command input is segmented. A basis function framework is developed and applied in $[8,34,54]$ using polynomial basis functions, which is further extended to rational basis functions in [61]. These basis functions are typically selected based on prior information, e.g., based on the approach in [31], and trial-and-error.

In model estimation and signal processing, the use of measured signals has comparable consequences, which has led to new regularization-based approaches that enforce sparsity. Early approaches include the non-negative garrote $[10]$ and Least Absolute Shrinkage and Selection Operator (LASSO) [50]. These are further generalized in $[3,13,25,52]$. Related applications in system identification include $[38,46]$.

Although important developments have been made in ILC and several successful applications have been reported, present approaches do not yet exploit the potential of enforcing additional structure and sparsity. The aim of the present paper is to develop a unified optimization-based approach to ILC that allows for explicitly enforcing structure and sparsity, enabling improved resource efficiency, disturbance attenuation, and flexibility to varying reference signals. The approach employs convex relaxations, enabling the use of standard optimization routines.

The main contribution of the present paper is a unified framework to sparse ILC. As subcontributions, trial-varying disturbances are analyzed in detail for explicit ILC algorithms (Section 3). Subsequently, a general optimization-based framework to sparse ILC is developed (Section 4), including many specific cases that are relevant to ILC applications. The results are confirmed through an application to a wafer stage system (Section 5). Related developments to the results in the present paper include the use of sparsity in control, where the main results have been related to Model Predictive Control (MPC), see $[2,21,29]$.

Notation: Throughout, $\|x\|_{\ell_{p}}$ denotes the usual $\ell_{p}$ norm, $p \in \mathbb{Z}_{>0}$. Also, $\|x\|_{0}=\sum_{i} \mathbf{1}\left(x_{i} \neq 0\right)$, i.e., the cardinality of $x$. Note that $\|x\|_{0}$ is not a norm, since it does not satisfy the homogeneity property. It relates to the general $\ell_{p}$ norm by considering the limit $p \rightarrow 0$ of $\|x\|_{p}$. A signal $x \in \mathbb{R}^{N}$ is called sparse if many of its components are zero, in which case $\|x\|_{0} \ll N$. In addition, $\|\tilde{X}\|_{\mathcal{L}_{\infty}}$ and $\|\tilde{X}\|_{\mathcal{H}_{\infty}}$ denote the usual $\mathcal{L}_{\infty}$ and $\mathcal{H}_{\infty}$ norms of discrete time systems, respectively. Throughout, $J$ denotes a system that maps an input space to an output space, operating either over finite or infinite time, which follows from the context. In certain cases, the system is assumed linear, time invariant, and scalar, with transfer function representation $\tilde{J}$. The power spectrum of a signal $x$ is denoted $\phi_{x}$, and is defined as in [32, Section 2.3].

\section{Problem formulation}

Consider the ILC system

$e_{j}=r-J f_{j}-v_{j}$

be given, where $e_{j} \in \ell_{2}$ denotes the error signal to be minimized, $r \in \ell_{2}$ is the reference signal, $f_{j} \in \ell_{2}$ denotes the command signal, and $v_{j} \in \ell_{2}$ represents trial-varying disturbances, including measurement noise. Here and in the sequel, all signals are tacitly assumed to have appropriate dimensions. Furthermore, $J$ represents the true system, either open-loop or closed-loop, with causal and stable transfer function $\tilde{J} \in \mathcal{R} \mathcal{H}_{\infty}$. The index $j \in \mathbb{Z}_{\geq 0}$ refers to the trial number. Throughout, the command signal $f_{j+1}$ is generated by an ILC algorithm

$f_{j+1}=F\left(f_{j}, e_{j}\right)$,

where the ILC update $F$ is defined in more detail later on. The general setup (1) encompasses the parallel ILC setup in Fig. 1, where

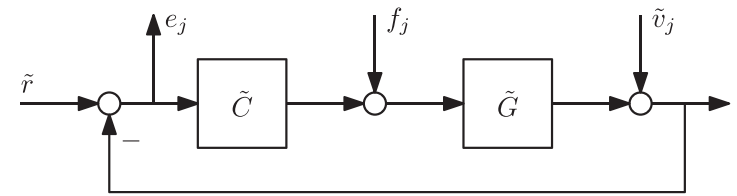

Fig. 1. Parallel ILC structure (3) as an example of (1).

$e_{j}=S \tilde{r}-S G f_{j}-S \tilde{v}_{j}$

where $S$ follows from its transfer function $\tilde{S}=\frac{1}{1+\tilde{G} \tilde{C}}, r=S \tilde{r}, J=S G$, $v_{j}=S \tilde{v}_{j}$, and $\tilde{C}, \tilde{G}$ are assumed to be linear.

From (2) and (1), it is immediate that the trial-varying disturbance $v_{j}$ directly affects the ILC command signal. In view of this observation, the problem investigated in this paper is to develop an ILC algorithm (2) that satisfies the following requirements:

R1) the iteration (1)-(2) is convergent over $j$;

R2) the iteration (1)-(2) leads to a small error $e_{j}$ in the presence of trial-invariant disturbances $r$ and trial-variant disturbances $v_{j}$;

R3) the resulting command signal $f_{j}$ has a certain structure, including

(a) a small $\left\|f_{j}\right\|_{0}$, and/or,

(b) a piecewise constant $f_{j}$ with a small number of jumps.

Here, R1 is a basic requirement for any ILC algorithm and ensures stability in the trial domain, in addition to the assumed stability in the time domain that is guaranteed by stability of $J$ in (1), see also [45] for the stability of such two-dimensional systems. Requirement R2 essentially states that the ILC algorithm should effectively compensate for $r$, while avoiding amplification of trial-varying disturbances $v_{j}$. Requirement R3 is imposed to enable resource-efficient implementations in terms of sampling or communication requirements, depending on the particular application requirements.

\section{Analysis of trial-varying disturbances in explicit ILC}

The main contribution of this paper is a design framework for ILC that allows for enforcing sparsity and structure in the command signals, which in turn allows for explicitly addressing aspects (i)-(iii) as mentioned in Section 1. The main mechanism behind these aspects (i)-(iii) are trial-varying disturbances. First, ILC uses measured data, where trial-varying disturbances lead to highly unstructured command signals, which in turn are expensive to implement. Second, these trial-varying disturbances are amplified by typical ILC algorithms. Third, typical ILC algorithms are inflexible to changing reference signals, which can in fact be interpreted as trial-varying disturbances. Since trial-varying disturbances have a central role in all these aspects (i)-(iii), these trial-varying references are analyzed in typical ILC algorithms in this section.

In particular, explicit linear ILC algorithms of the general form

$f_{j+1}=Q\left(f_{j}+L e_{j}\right)$

are considered. The infinite time scalar case is considered, where $Q: \ell_{2} \mapsto \ell_{2}$ and $L: \ell_{2} \mapsto \ell_{2}$. Here, $Q$ and $L$ have associated transfer functions $\tilde{Q} \in \mathcal{R} \mathcal{L}_{\infty}$ and $\tilde{L} \in \mathcal{R} \mathcal{L}_{\infty}$. Note that $\tilde{J} \in \mathcal{R} \mathcal{H}_{\infty}$ reflects causality and stability of the system. The fact that $\tilde{Q} \in \mathcal{R} \mathcal{L}_{\infty}$ and $\tilde{L} \in \mathcal{R} \mathcal{L}_{\infty}$ reflects that typical ILC algorithms are typically noncausal, and are usually implemented such that bounded solutions are obtained through finite-time preview or via stable inversion through a bilateral Z-transform [60].

The trial-varying disturbance $v_{j}$ in (1) will propagate throughout the iterations through the iteration-domain update (4). The following assumption is widely adopted [32]. 
Assumption 1. Let $v_{j}=H n_{j}$, where $n_{j}$ is i.i.d. zero-mean white noise with variance $\lambda_{e}, \tilde{H}$ monic and bistable.

Clearly, $v_{j}$ typically does not have compact support. As a result, $f_{j+1}$ will not have compact support in general due to ILC algorithm (4).

To enable a more detailed analysis, the following auxiliary result provides a suitable condition to guarantee that the iteration defined by (1) and (4) converges.

Theorem 2. The iteration defined by (1)-(4) converges monotonically in the $\ell_{2}$ norm to a fixed point $f_{\infty}$ and resulting $e_{\infty}$ iff

$\|\tilde{Q}(1-\tilde{L} \tilde{J})\|_{\mathcal{L}_{\infty}}<1$.

Proof. Substituting (1) into (4) leads to

$f_{j+1}=Q(I-L J) f_{j}+Q L r-Q L v_{j}$.

Using transfer function representations and subsequent application of the Banach fixed-point theorem in conjunction with [57, Theorem 4.4] yields the desired result.

Note that Theorem 2 allows for non-causal ILC algorithms, i.e., $Q, L \in \mathcal{R} \mathcal{L}_{\infty}$. This is more general compared to related analyses, including [35, Chapter 3], which only allow for causal ILC algorithms by restricting to the $\mathcal{H}_{\infty}$ norm.

The following result is the main result of this section and reveals the propagation of noise in the iteration defined by (1) and (4).

Theorem 3. Given the system (1) and ILC update (4) with $f_{0}=0$, Assumption 1, and that the iteration is stable in the sense of Theorem 2 , then the spectrum of $e_{\infty}$ is given by

$\phi_{e_{\infty}}=\left|\frac{1-\tilde{Q}}{1-\tilde{Q}(1-\tilde{L} \tilde{J})}\right|^{2} \phi_{r}+\left(1+\frac{|\tilde{J} \tilde{Q} \tilde{L}|^{2}}{1-\mid \tilde{Q}\left(1-\left.\tilde{L} \tilde{J}\right|^{2}\right.}\right) \phi_{v}$.

Theorem 3 provides a detailed analysis of the propagation of noise for the general ILC algorithm (4). In special cases, the result can be further simplified. For instance, in inverse-model ILC, $\tilde{Q}=1$ and $\tilde{L}=\tilde{J}^{-1} \in \mathcal{R H} \mathcal{H}_{\infty}$, in which case Theorem 3 reveals that

$\phi_{e_{\infty}}=2 \phi_{v}$.

The result (6) reveals that the limit error spectrum involves an amplification of the noise spectrum by a factor of two.

Inclusion of a learning gain $\alpha \in(0,1]$ in inverse-model ILC, i.e., replacing (4) by $f_{j+1}=Q\left(f_{j}+\alpha L e_{j}\right)$, mitigates the amplification of trial-varying disturbances, i.e.,

$\phi_{e_{\infty}}=\left(1+\frac{\alpha^{2}}{2 \alpha-\alpha^{2}}\right) \phi_{v}$.

By taking $\alpha \rightarrow 0$, a first-order Taylor series approximation yields

$\phi_{e_{\infty}} \approx\left(1+\frac{1}{2} \alpha\right) \phi_{v}$

Hence, by choosing $\alpha$ nonzero and small leads to a limit error $\phi_{e_{\infty}}=\phi_{v}$, which intuitively corresponds to the optimal result, since the iteration-domain feedback (4) cannot attenuate $v_{j}$ in iteration $j$. Note that $\alpha=0$ turns of learning, in which case the trialinvariant error due to $r$ is not compensated for. An alternative to attenuate $\phi_{v}$ is to re-design the controller $C$ in (3), which should from a disturbance attenuation perspective be designed such that $\tilde{S} \approx \tilde{H}^{-1}$, as is advocated in [6]. Note that this affects $J$ in (1).

Remark 4. The results in this section rely on infinite time signals and LTI systems. Alternative ILC designs based on finite-time optimization [12], see also the forthcoming section, explicitly address the boundary effects, typically leading to an LTV ILC update (2), even if $J$ is LTI. In [60], it is shown that these optimization-based designs are equivalent to a certain linear-quadratic-tracking problem. As a result, the solution reaches a certain stationary value for sufficiently long task lengths, in which case an LTI $L$ and $Q$ can be derived for which the results of Theorem 3 apply. This also implies that the design of weighting filters for such optimization-based design can be further investigated using the results presented in this section, as is briefly summarized in the next section.

\section{Sparse ILC}

In this section, the general optimization-based ILC framework is presented that allows for enforcing additional structure compared to alternative ILC structure. In fact, traditional norm-optimal ILC algorithms [12] are recovered as a special case. In the next subsection, the general framework is presented and motivated, followed by specific design choices in the subsequent sections.

\subsection{General approach}

Throughout, the criterion

$$
\begin{aligned}
\mathcal{J}\left(f_{j+1}\right)= & \frac{1}{2}\left\|W_{e} e_{j+1}\right\|_{2}^{2}+\frac{1}{2}\left\|W_{f} f_{j+1}\right\|_{2}^{2} \\
& +\frac{1}{2}\left\|W_{\Delta f}\left(f_{j+1}-f_{j}\right)\right\|_{2}^{2}+\lambda\left\|D f_{j+1}\right\|_{1}
\end{aligned}
$$

is considered. Here, finite time signals of length $N$ are considered to obtain an optimization problem with a finite number of decision variables, i.e., $e_{j}, f_{j} \in \mathbb{R}^{N}$. The matrices are defined in the sequel and are assumed to have compatible dimensions. In addition, existence of a unique solution is typically assumed, which can be directly enforced by assuming appropriate positive (semi-) definiteness assumptions on the design variables $W_{e}, W_{f}, W_{\Delta f}, D$, and $\lambda$. Also, $e_{j+1}$ in (8) is considered to be the noise-free prediction $e_{j+1}=r-J f_{j+1}$. Since also $r$ is unknown, the main idea in ILC is to use this approximation also for $e_{j}$, leading to

$e_{j+1}=e_{j}-J\left(f_{j+1}-f_{j}\right)$,

where $e_{j}$ is the measured error signal during trial $j$. Thus, substituting (9) into (8) renders the optimization problem as a function of the known variables $e_{j}, f_{j}$, user-defined variables, and the decision variable $f_{j+1}$.

The motivation for considering (8) is as follows. First, if $\lambda=0$, then standard norm-optimal ILC is recovered, e.g., as in [23]. In this case, an analytic solution of the form (4) is directly obtained with

$L=\left(J^{T} \bar{W}_{e} J+W_{\Delta f}\right)^{-1} J^{T} \bar{W}_{e}$

$Q=\left(J^{T} \bar{W}_{e} J+\bar{W}_{f}+\bar{W}_{\Delta f}\right)^{-1}\left(J^{T} \bar{W}_{e} J+\bar{W}_{\Delta f}\right)$,

where $\bar{W}_{e}=W_{e}^{T} W_{e}, \bar{W}_{f}=W_{f}^{T} W_{f}$, and $\bar{W}_{\Delta f}=W_{\Delta f}^{T} W_{\Delta f}$.

The second motivation stems from the observation that the terms $\frac{1}{2}\left\|W_{f} f_{j+1}\right\|_{2}^{2}$ and $\frac{1}{2}\left\|W_{\Delta f}\left(f_{j+1}-f_{j}\right)\right\|_{2}^{2}$ essentially involve a ridge regression or Tikhonov regularization. If $f_{j}=0$, then the two terms coincide. If $f_{j} \neq 0$, i.e., during the ILC iterations, then $W_{f}$ typically leads to $Q \neq I$ in (4), providing robustness with respect to modeling errors [11]. Increasing $W_{\Delta f}$ attenuates trial-varying disturbances, which is similar to reducing $\alpha$ in (7). Note that $W_{f}$ penalizes the command input and hence to a certain extent mitigates the attenuation of trial-varying disturbances. However, it is highly recommended to use $W_{\Delta f}$ to decrease trial-varying disturbances, since the use of $W_{f}$ leads to a non-zero limit error $e_{\infty}$ in the presence of trial-invariant disturbances, which coincides with a $\tilde{Q} \neq 1$ in Theorem 3. 


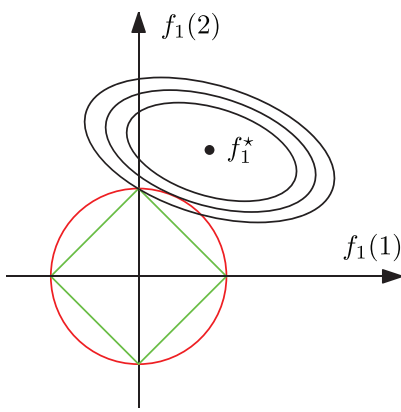

Fig. 2. Enforcing sparsity in ILC. Assuming $N=2, f_{1}$ contains two elements. The constraint set, i.e., the $\ell_{1}$ ball is plotted in green. In addition, ellipsoidal contour lines corresponding to the objective in (13) are plotted. The optimal solution is found at the point where the contour line first touches the constraint set, which in this case implies $f_{1}(1)=0$, hence $f_{1}$ is sparse. In contrast, in the ridge regression case of (15) (whose constraint is shown in red), the solution is not sparse. In particular, this solution is obtained when the contour lines of the objective function in (15) first touches the constraint set corresponding to the $\ell_{2}$ ball. In addition, $f_{1}^{\star}$ denotes the unconstrained solution to the objective function in (13) and (15). (For interpretation of the references to colour in this figure legend, the reader is referred to the web version of this article.)

The third and main motivation for considering the extended criterion (8) is the additional term $\lambda\left\|D f_{j}\right\|_{1}$ that is used to enforce sparsity and structure. Note that sparsity is measured directly through the $\ell_{0}$ norm. However, inclusion of an $\ell_{0}$ penalty in the criterion (8) leads to a non-convex optimization problem, which in fact is NP-hard, see [37]. The $\ell_{1}$ norm is a convex relaxation of the $\ell_{0}$ norm. To see this, note that (8) is essentially in Lagrangian form. For the purpose of explanation, consider the simplified form by selecting $W_{e}=I, j=1, f_{0}=0, W_{f}=0, W_{\Delta f}=0$, and $D=I$. Using (9)

$\mathcal{J}\left(f_{1}\right)=\frac{1}{2}\left\|e_{0}-J f_{1}\right\|_{2}^{2}+\lambda\left\|f_{1}\right\|_{1}$,

which is equivalent to the primal optimization problem

$\begin{array}{ll}\min _{f_{1}} & \frac{1}{2}\left\|e_{0}-J f_{1}\right\|_{2}^{2} \\ \text { subject to } & \left\|f_{1}\right\|_{1} \leq t .\end{array}$

for the range of $t$ where the constraint in (13) is active. This implies that for a given value of $\lambda$, there exists a value of $t$ for which (12) and (13) have identical minima. In this simplified case, the interpretation in [50] applies to the ILC problem. In particular, the constraint in (13) is plotted in Fig. 2 in addition to several elliptical contour lines of the objective function in (13). The solution to (13) corresponds to the smallest ellipsoid that touches the rhombus of the constraint. If this happens at the corner, as is the case in Fig. 2, then one of the coefficients is zero and a sparse solution is obtained.

In contrast, traditional norm-optimal ILC, i.e., corresponding to the solution (10)-(11), typically does not lead to a sparse solution with zero entries in $f_{1}$. To see this, consider a similar simplified case as in (12)

$\mathcal{J}\left(f_{1}\right)=\frac{1}{2}\left\|e_{0}-J f_{1}\right\|_{2}^{2}+\tau\left\|f_{1}\right\|_{2}$,

which is again in Lagrangian form. Here, $\tau$ directly relates to the weights in (8) if $W_{f}$ and $W_{\Delta f}$ are selected as the common diagonal case with initialization $f_{0}=0$. The primal optimization problem corresponding to (14) is given by

$\begin{array}{ll}\min _{f_{1}} & \frac{1}{2}\left\|e_{0}-J f_{1}\right\|_{2}^{2} \\ \text { subject to } & \left\|f_{1}\right\|_{2} \leq t .\end{array}$

In Fig. 2, the constraint is again shown together with the contour lines of the objective function. Due to the lack of corners of the constraint in this two-dimensional example, zeros only occur when the principal axes of the elliptical contour lines of the objective functions coincide with the axes of the command input. Hence, the $\ell_{1}$ norm promotes sparse solutions, whereas the $\ell_{2}$ norm in general does not.

The results in Fig. 2 involve a two-dimensional example. From this example, it can also be observed why the $\ell_{1}$ norm is chosen as a surrogate of the $\ell_{0}$ norm, because it is the convex envelope of the $\ell_{0}$ norm in the $\ell_{2}$ ball, i.e., it is the largest convex function whose graph lies below that of the $\ell_{0}$ norm in the set $\left\|f_{1}\right\|_{2} \leq 1$.

A similar mechanism takes place in higher dimensions. Indeed, the $\ell_{1}$ ball, in any finite number of dimensions, can be shown to be a polytope, i.e., described by a set of linear inequalities. In this case, the corners in Fig. 2 correspond to extreme points, which is a 0 -dimensional face. Here, a face is subset of $F$ of a convex set such that if $f_{1}$ is in $F$ and $f_{1}=a y+(1-a) z$, with $y$ and $z$ in the convex set and $a \in[0,1]$, then $a$ should be equal to 0 or 1 . In the $\ell_{1}$ ball, the faces correspond to sparse sets, i.e., to points most of whose entries are 0 , so when the ellipsoid intercepts one of these faces, the solution is typically highly sparse. The higher dimensional case is further investigated in $[13,17,50]$.

Finally, it is remarked that if $\lambda>0$, then the solution to (8) typically cannot be obtained in closed-form as in (10)-(11). Interestingly, a unique solution to (8) exists due to convexity. The optimization problem (8) can be readily solved using general convex optimizers. In addition, several efficient algorithms have been developed, see, e.g., [25, Chapter 5] for an overview. Several of such algorithms provide the entire solution path as a function of $\lambda$. The particular algorithm depends on the choice of $D$, but several relevant choices are outlined below.

\subsection{Sparse command signals via lasso}

In view of requirement $\mathrm{R} 3 \mathrm{a}$ in Section 2, in certain applications it is required to have a sparse command signal $f_{j}$. To this end, $D$ in (8) can be selected as $D=I$. As a result, the value of $\lambda>0$ will dictate the sparsity of the solution. In addition, in this classical lasso approach, $W_{f}$ and $W_{\Delta f}$ may be selected as $W_{f}=0$ and $W_{\Delta f}=0$, i.e., traditional design guidelines for norm-optimal ILC regarding positive definiteness of these matrices, as in [23], need not be considered, even for the situation where $J$ is singular. The resulting criterion becomes

$\mathcal{J}\left(f_{j+1}\right)=\frac{1}{2}\left\|W_{e}\left(e_{j}-J f_{j+1}\right)\right\|_{2}^{2}+\lambda\left\|f_{j+1}\right\|_{1}$,

which closely reflects the original lasso approach in [50].

\subsection{Elastic net lasso}

In the lasso ILC approach in Section 4.2, the commonly used weighting matrices in $W_{f}$ and $W_{\Delta f}$ are set to zero. Interestingly, by selecting either $W_{f}$ or $W_{\Delta f}$ unequal to zero, an ILC algorithm that relates to the elastic net is obtained, see [59], which combines lasso and ridge regression. An important advantage is that the elastic net improves group sparsity, where several components become zero simultaneously. Notice that a drawback of the socalled naive elastic net, which coincides with $W_{f} \neq 0, W_{\Delta f}=0$, is that it leads to a double shrinkage, and it benefits from a correction step [59]. In contrast, in ILC the alternative choice $W_{f}=0$, $W_{\Delta f} \neq 0$ can be made, which enforces sparsity in addition to attenuating trial-varying disturbances, see Section 4.1. 


\subsection{Sparse updates via fused lasso}

In view of Requirement R3b, it may be required that the signal $f_{j}$ is not necessarily sparse but piecewise constant, i.e., its value is only changed occasionally in time. This requires a certain structure of signal, which is different than sparsity $\left\|f_{j}\right\|_{0}$. The main idea is to select $D$ as

$D_{f}=\left[\begin{array}{ccccc}-1 & 1 & & & \\ & -1 & 1 & & \\ & & \ddots & \ddots & \\ & & & -1 & 1\end{array}\right]$,

a choice which is also known as the fused lasso, e.g., [51] and leads to the criterion

$\mathcal{J}\left(f_{j+1}\right)=\frac{1}{2}\left\|W_{e}\left(e_{j}-J f_{j+1}\right)\right\|_{2}^{2}+\lambda\left\|D_{f} f_{j+1}\right\|_{1}$.

Interestingly, the fused lasso (18) can be recast as a traditional lasso of the form (16), yet with an increment-input-output system description. To establish the connection, let $\tilde{J}^{i}=\tilde{J}\left(1-z^{-1}\right)$ be the increment-input-output system. Also, expand $D_{f}$ in (17) as

$D_{f}^{i}=\left[\begin{array}{ccccc}1 & & & & \\ -1 & 1 & & & \\ & -1 & 1 & & \\ & & \ddots & \ddots & \\ & & & -1 & 1\end{array}\right]$.

Then, a change of variables

$f_{j+1}^{i}=D_{f}^{i} f_{j+1}$,

where $f_{j+1}^{i}$ denotes the incremental input, leads to

$\mathcal{J}\left(f_{j+1}\right)=\frac{1}{2}\left\|W_{e}\left(e_{j}-J^{i} f_{j+1}^{i}\right)\right\|_{2}^{2}+\lambda\left\|f_{j+1}^{i}\right\|_{1}$,

with $J^{i}=J\left(D_{f}^{i}\right)^{-1}$ corresponding to $\tilde{J}^{i}$.

\subsection{Sparse fused lasso}

Up to this point, Requirement R3a and Requirement R3b have been addressed separately in Section 4.2 and Section 4.4, respectively. In certain applications, it may be desired to impose both Requirement R3a and Requirement R3b.

Interestingly, Requirement R3a and Requirement R3b can be enforced both by selecting

$D=\left[\begin{array}{ll}\alpha D_{f} & I\end{array}\right]$,

in (8). Here, the parameter $\lambda$ can still be chosen to enforce sparsity, i.e., Requirement R3a, whereas the additional tuning parameter $\alpha \in$ $\mathbb{R}_{\geq 0}$ enforces Requirement R3b. This leads to the so-called sparse fused lasso [52]. Note that additional requirements can easily be incorporated using a similar construction as (19).

\subsection{Basis function ILC}

In recent extensions to ILC, several basis functions are employed. On the one hand, wavelet basis functions are used in, e.g., [33]. These immediately fit in the formulation (8), see also [52, Section 2.1.3], enabling a systematic way for thresholding while explicitly addressing the performance criterion.

On the other hand, flexibility to varying reference signals is achieved by employing basis functions that depend on the reference. In particular, the command signal is parameterized as $f_{j+1}=$ $\Psi(r) \theta_{j+1}$, see, e.g., $[8,34,54,61]$. The proposed framework can be employed to minimize the number of required basis functions. For instance, a large set can be postulated, e.g., following the guidelines in [31]. Next, an alternative formulation of (8) can be considered, e.g.,

$$
\begin{array}{ll}
\min _{\theta_{j+1}} & \left\|\theta_{j+1}\right\|_{1} \\
\text { subject to } & \frac{1}{2}\left\|W_{e} e_{j+1}\right\|_{2}^{2}+\frac{1}{2}\left\|W_{f} \Psi(r) \theta_{j+1}\right\|_{2}^{2} \\
& +\frac{1}{2}\left\|W_{\Delta f} \Psi(r)\left(\theta_{j+1}-\theta_{j}\right)\right\|_{2}^{2} \leq t,
\end{array}
$$

where a suitable value of $t$ can be obtained by solving the standard norm-optimal ILC in (10)-(11).

\subsection{Extensions, analysis, and discussion}

A general framework for enforcing sparsity and structure in iterative learning control has been proposed, and several specific choices have been outlined. The main idea is that the proposed framework, involving criterion (8), leads to a command signal $f_{j}$ that is either sparse, i.e., $\left\|f_{j}\right\|_{0}$ is small, or has structure, i.e., a piecewise constant $f_{j}$ with a small number of jumps. This is achieved by the lasso and fused lasso, respectively. This structure has several important benefits. First, this leads to a reduced implementation cost in terms of sampling or communication requirements due to highly structured command signals. Second, it substantially reduces the amplification of trial-varying disturbances. From the perspective of ILC, the proposed sparse ILC framework essentially generates basis functions [54], which becomes explicit in Section 4.7.1. In turn, these weighting filters enable which leads to a reduction of trial-varying disturbances [8]. Also, from Bolder et al. [8], it is clear that the basis has to be sufficiently rich, which is investigated in more detail in Section 4.7.2. Third, this idea of automated basis function selection can also be combined with earlier ideas on using basis functions, i.e., to select them from a prespecified library $\Psi(r)$, see Section 4.6.

From another perspective, the use of sparsity can also be seen as a time-domain filtering operation. Indeed, in typical ILC designs, frequency-domain specifications are used, e.g., to account for trial-varying disturbances, see $[42,48]$. This also relates to the idea of the static learning gain $\alpha$ in (7). The proposed framework essentially provides a learning gain $\alpha$ that turns off learning in regions where it does not contribute significantly to attenuating trial-invariant disturbances, in view of not amplifying trial-varying disturbances.

Further extensions that are beyond the scope of the present paper but can be directly incorporated include group lasso [56], adaptive lasso [58], reweighted $\ell_{1}$ [16], and the use of non-convex penalties [4].

\subsubsection{Re-estimation for debiasing}

Note that the lasso shrinks the estimate compared to the leastsquares terms in (8). Through a re-estimation step of the nonzero coefficients, debiasing is obtained. Note that in certain cases, the bias helps to obtain a smaller overall error, i.e., including both bias and variance aspects, which closely relates to the well-known Stein estimator [49]. However, for ILC such a bias is undesired, since it is automatically eliminated by performing iterations, see Theorem 2 . Thus, it is expected that as the ILC iterations increase, the advantages of re-estimating for debiasing become more important. Similar re-estimation steps are proposed in [46,47], [36, Page 439], [30, Section 7.1]. Interestingly, in the context of ILC, the idea of enforcing sparsity followed by a re-estimation step essentially has the same role as a $Q$-filter in traditional ILC, see [5] for details. Finally, it is pointed out that the re-estimation essentially involves a standard norm-optimal ILC design, i.e., (8) with $\lambda=0$, in conjunction with a command input $f_{j+1}$ with reduced dimension. These can in 
fact be solved efficiently, i.e., linearly in the dimension of the reduced command input, see [60] for details.

\subsubsection{Sparse signal recovery}

The main motivation for using the $\ell_{1}$ norm in (8) essentially is to provide a convex relaxation of the $\ell_{0}$ norm. In case the optimal command input, i.e., for $j \rightarrow \infty$ and $v_{j}=0$, the signal $f_{\infty}$ that minimizes $\mathcal{J}\left(f_{\infty}\right)$, is sparse, a relevant question is whether this optimal sparse vector can be recovered using the formulation (8). The answer depends on the sparsity of the underlying optimal command input $f_{j}$, as well as on the matrix $J$. In [17], a sufficient condition that relies on the restricted isometry property is provided. However, these conditions are violated for many practical cases. Nonetheless, the formulation (8) provides an effective way to enforce sparsity.

\subsubsection{Monotonic convergence}

Monotonic convergence is a commonly used requirement for practical applications. Indeed, it is well-known that poorly designed ILC algorithms can lead to a significant learning transient. It is well-known that traditional norm-optimal ILC, i.e., setting $\lambda=0$ in (8), is monotonically convergent in $f_{j}$, see, e.g., [11], where the usual assumption $v_{j}=0$ is tacitly assumed to analyze monotonic convergence. However, if $\lambda>0$, the criterion (8) involves multiple norms, i.e., both the $\ell_{1}$ and the $\ell_{2}$ norm. As a result, monotonic convergence requires a more detailed analysis.

To proceed, consider for instance the elastic net lasso of Section 4.3 with $D=I, W_{f}=0, W_{\Delta f} \succ 0$. In this case, monotonic convergence of the ILC cannot be guaranteed in general if $\lambda=0$. Interestingly, in this case the criterion (8) can be recast as

$$
\begin{aligned}
\mathcal{J}\left(f_{j+1}\right)= & \frac{1}{2}\left\|\left(\left[\begin{array}{c}
W_{e} e_{j} \\
0
\end{array}\right]+\left[\begin{array}{c}
W_{e} \\
W_{\Delta f}
\end{array}\right] f_{j}\right)-\left[\begin{array}{c}
W_{e} \\
W_{\Delta f}
\end{array}\right] f_{j+1}\right\|_{2}^{2} \\
& +\lambda\left\|f_{j+1}\right\|_{1} .
\end{aligned}
$$

Next, there exists a value of $\tau$ such that the optimization problem

$$
\begin{array}{ll}
\min _{f_{j+1}} & \left\|f_{j+1}\right\|_{1} \\
\text { subject to } & \frac{1}{2}\left\|\left[\begin{array}{c}
W_{e} e_{j} \\
0
\end{array}\right]-\left[\begin{array}{c}
W_{e} \\
W_{\Delta f}
\end{array}\right]\left(f_{j+1}-f_{j}\right)\right\|_{2}^{2} \leq \tau .
\end{array}
$$

has an identical solution as (20) at a certain iteration $j$. By fixing $\tau$ during the iterations, the criterion (22) can be directly used to enforce monotonic convergence of $f_{j}$ in the $\ell_{1}$ norm. Alternatively, the formulation

$$
\min _{f_{j+1}} \quad \frac{1}{2}\left\|\left[\begin{array}{c}
W_{e} e_{j} \\
0
\end{array}\right]-\left[\begin{array}{c}
W_{e} \\
W_{\Delta f}
\end{array}\right]\left(f_{j+1}-f_{j}\right)\right\|_{2}^{2}
$$

\section{subject to $\left\|f_{j+1}\right\|_{1} \leq \beta$}

can be considered for a fixed $\beta$, which enforces monotonic convergence in the $\ell_{2}$ norm.

\section{Application to a wafer stage}

\subsection{Setup}

The considered system is a wafer stage, see Fig. 3. Wafer stages are positioning systems that are used in the production of integrated circuits (ICs) through a photolithographic process. The considered wafer stage is controlled in all six motion degrees-offreedom, i.e., three translations and three rotations. The system is a dual-stage system, where the long stroke enables a stroke of $1 \mathrm{~m}$ in

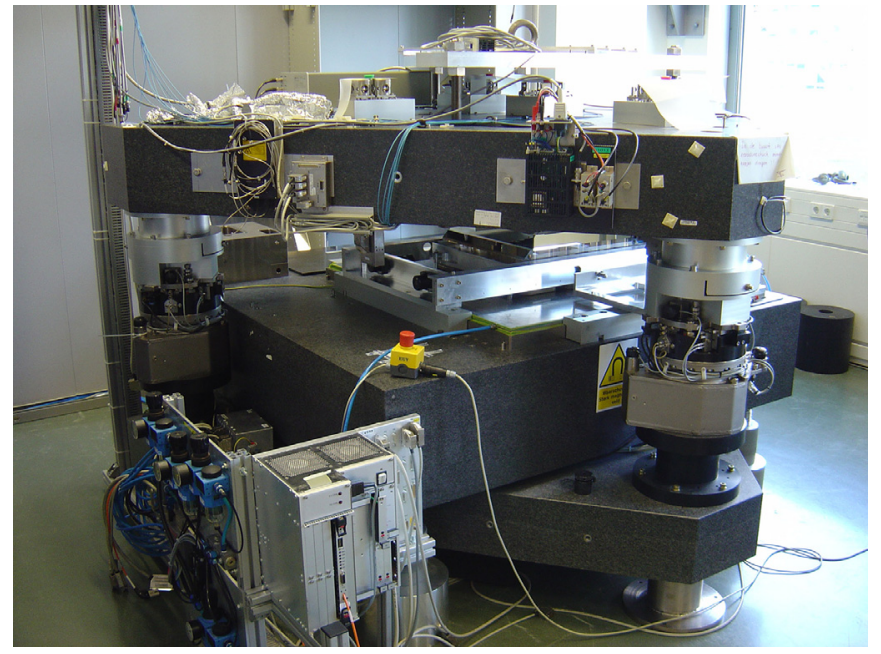

Fig. 3. Considered wafer stage application.

the horizontal plane, whereas the short stroke enables a positioning accuracy of $1 \mathrm{~nm}$. Further details on the system and the considered actuation and sensor system are provided in [40]. Throughout, a sampling frequency of $1 \mathrm{kHz}$ is adopted, as in [39].

To enable a detailed comparison between the various approaches in Section 4, the identified model in [39] is considered as true system, i.e., the result as described in [39] is denoted $G_{0}$. In addition, the feedback controller designed in [39] is adopted to stabilize the system. In Fig. 4, the open-loop $G_{0}$ and closed-loop $S_{0} G_{0}$ are depicted. In addition, a closed-loop model is made, where a model error is introduced by selecting $J=0.7 S_{0} G_{0}$. This model error is introduced to investigate robust convergence properties of ILC. The resulting model $J$ is also depicted in Fig. 4.

The additive noise $\tilde{v}_{j}$ is zero mean white noise with a normal distribution and variance $\lambda_{e}=1.5 \cdot 10^{-7}$. As a result, $H$ in Assumption 1 has transfer function $\tilde{H}=\frac{1}{1+\tilde{G} \tilde{C}}$.

The task $r$ is shown in Fig. 5, which is a position signal. In addition, the corresponding scaled acceleration profile is depicted, which is expected to constitute the main contribution of $f_{j}[31,34]$. For the considered wafer stage application in Fig. 3, the constant velocity phase is most important for performance, see [15, Figure 16 and Figure 20], which takes place between $0.03 \mathrm{~s}$ and $0.24 \mathrm{~s}$.

The goal of this section is to illustrate and compare the proposed approaches in Section 4. The reference situation, i.e., feedback only with $f_{0}=0$ in Fig. 1 is shown in Figs. 6 (solid blue), 7 , and 8. In particular, the approaches in Section 4 are applied in this section.

\subsection{Traditional norm-optimal ILC}

First, the traditional norm-optimal ILC solution is implemented with $\lambda=0$ in (8) with the analytic solution (10)-(11). Here, $W_{e}=I$, $W_{f}=0$, and $W_{\Delta f}=10^{-10}$. Notice that $W_{\Delta f}$ is relatively small but nonzero, since a nonzero $W_{\Delta f}$ or $W_{f}$ is required to enforce a unique optimal solution.

The results after 40 iterations are depicted in Fig. 6. Clearly, the error is reduced to a very small value. As is expected, the feedforward is nonzero at every time instant and very noisy.

To further analyze these results, the 2 norm of the stochastic, i.e., trial-varying, part of the error is computed as $\sqrt{\sum_{t=1}^{N}\left(e_{j}(t)-\hat{e}_{\infty}(t)\right)^{2}}$, see Fig. 7. Here, $\hat{e}_{\infty}$ is computed as

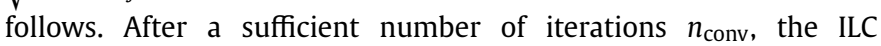
algorithm is assumed to have converged, after which an additional iterations $n_{\text {iter }}$ is used to compute $\hat{e}_{\infty}=\frac{1}{n_{\text {iter }}} \sum_{j=n_{\text {conv }}}^{n_{\text {conver }}+n_{\text {ite }}-1} e_{j}$. 

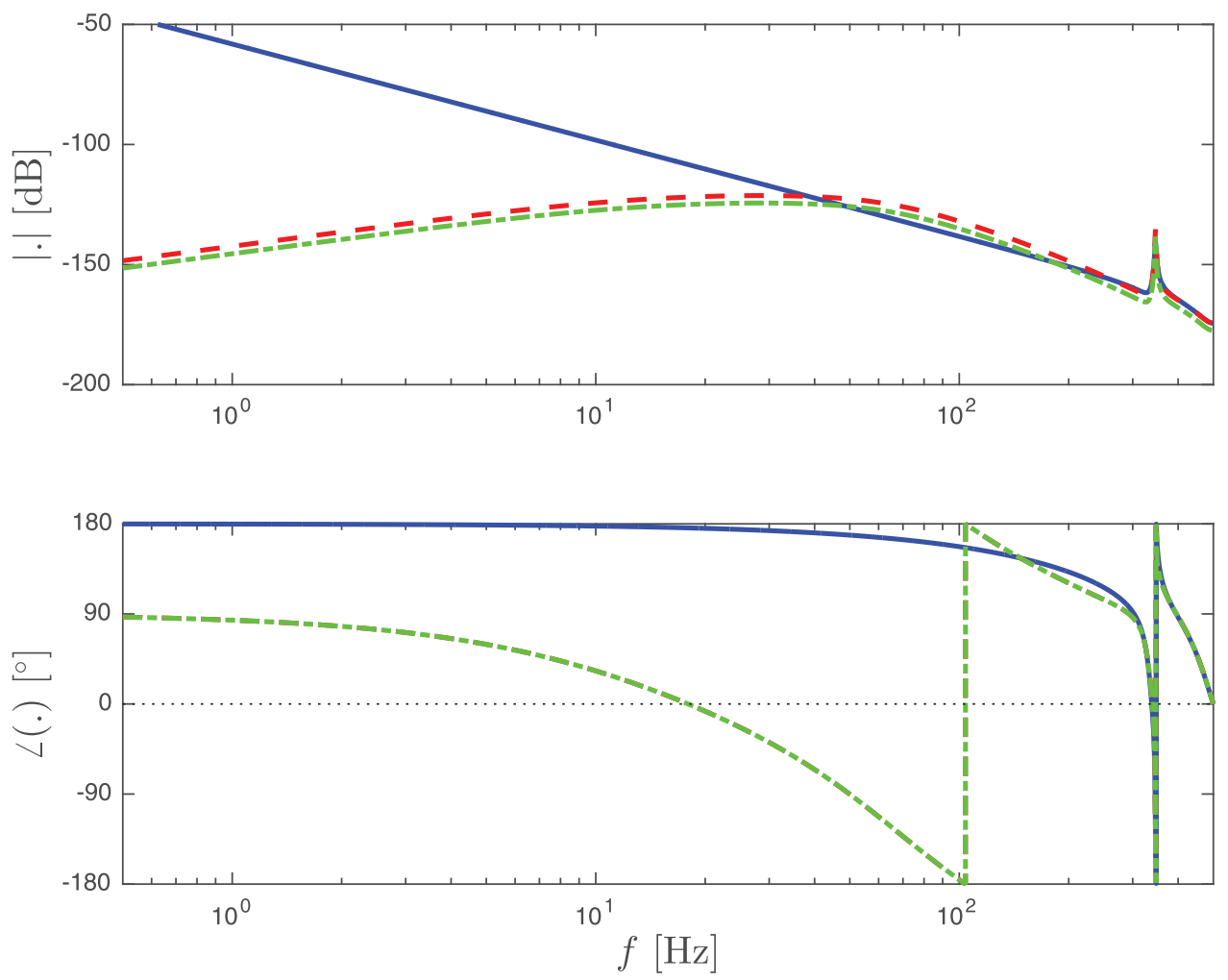

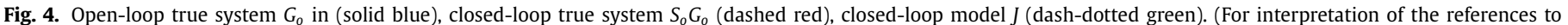
colour in this figure legend, the reader is referred to the web version of this article.)

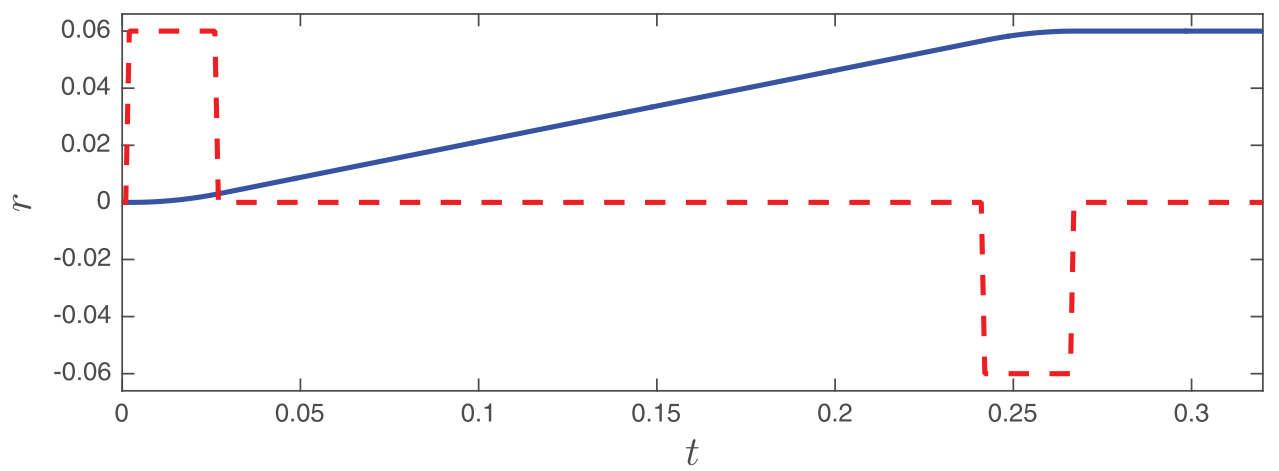

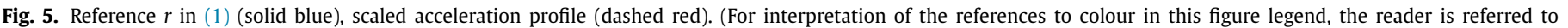
the web version of this article.)

Clearly, Fig. 7 reveals that the trial-varying part of the error is amplified by a factor 2 , which corroborates the result of Theorem 3 , where $Q \approx 1$ due to the specific selection of weighting filters.

To further investigate the amplification of trial-varying disturbances, the spectrum of the trial-varying part of the errors in Fig. 7 is estimated, see Fig. 8. In addition, the spectrum $\phi_{v}=$ $\left|\frac{1}{1+\widetilde{G} \widetilde{C}}\right|^{2} \lambda_{e}$ is computed, as well as $2 \phi_{v}$. Again, this clearly confirms the result of Theorem 3. In particular, the presented ILC approach with $\lambda=0$ and small $W_{f}$ and $W_{\Delta f}$ leads to a perfect attenuation of trial-invariant disturbances. However, it amplifies trial-varying disturbances by a factor two, and leads to an $f_{j}$ with large $\left\|f_{j}\right\|_{0}$, violating Requirement R3a, as well as R3b.

Summarizing, the results in Figs. 6-8 confirm that normoptimal ILC amplifies trial-varying disturbances, and leads to a non-sparse solution in view of Requirement R3a and Requirement R3b.

\subsection{Lasso ILC}

To address Requirement R3a, the approach in Section 4.2 is applied. In particular, $W_{e}=I, W_{f}=0$, and $W_{\Delta f}=0, D=I$, and $\lambda=$ $5 \cdot 10^{-9}$. Next, the ILC iteration is started, and after 40 iterations it leads to $e_{40}$ and $f_{40}$ in Fig. 11. Interestingly, $\left\|f_{40}\right\|_{0}$ is much smaller for the lasso ILC approach compared to the results of Section 5.2, as is confirmed in Fig. 9, thereby addressing Requirement R3a.

Also, the 2 norm of the error signal is computed, see Fig. 10. Clearly, the error reduces significantly over the iterations. Finally, also the re-estimated lasso, as is explained in Section 4.7, is implemented. The results are also depicted in Fig. 10. Interestingly, it can be observed that re-estimating leads to a smaller limit error, as is expected. However, note that during the iterations, the approach of Section 4.2 leads to a smaller error compared to the re-estimated version in several of the initial iterations. An explanation for this aspect is that the biased estimate leads to a smaller overall error, which is a similar effect as in the Stein estimator. Hence, it is con- 

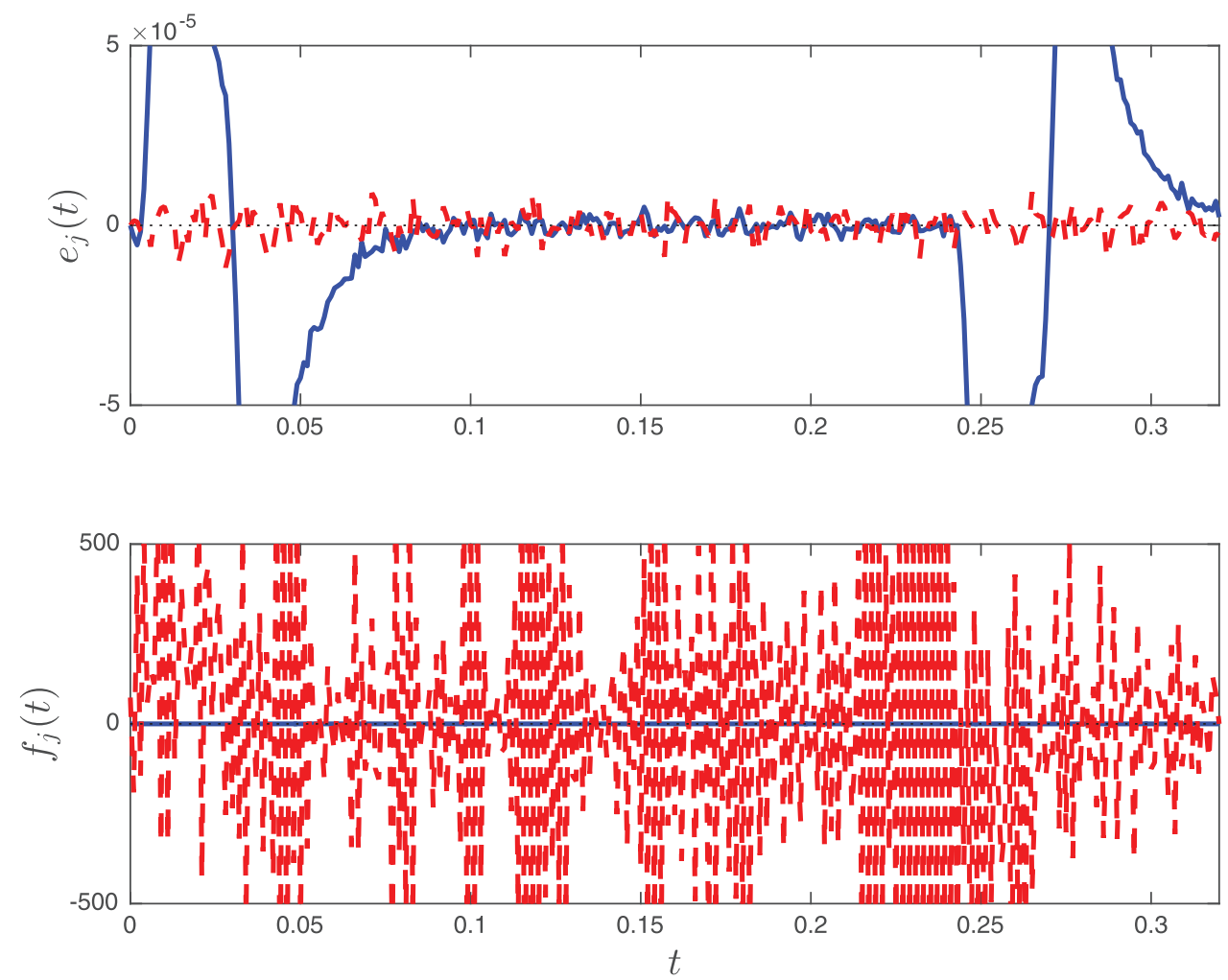

Fig. 6. Top: error $e_{j}$. Bottom: command signal $f_{j}$. Shown are iteration $j=0$ (solid blue), iteration $j=40$ for traditional norm-optimal ILC of Section 5.2 (dashed red). (For interpretation of the references to colour in this figure legend, the reader is referred to the web version of this article.)

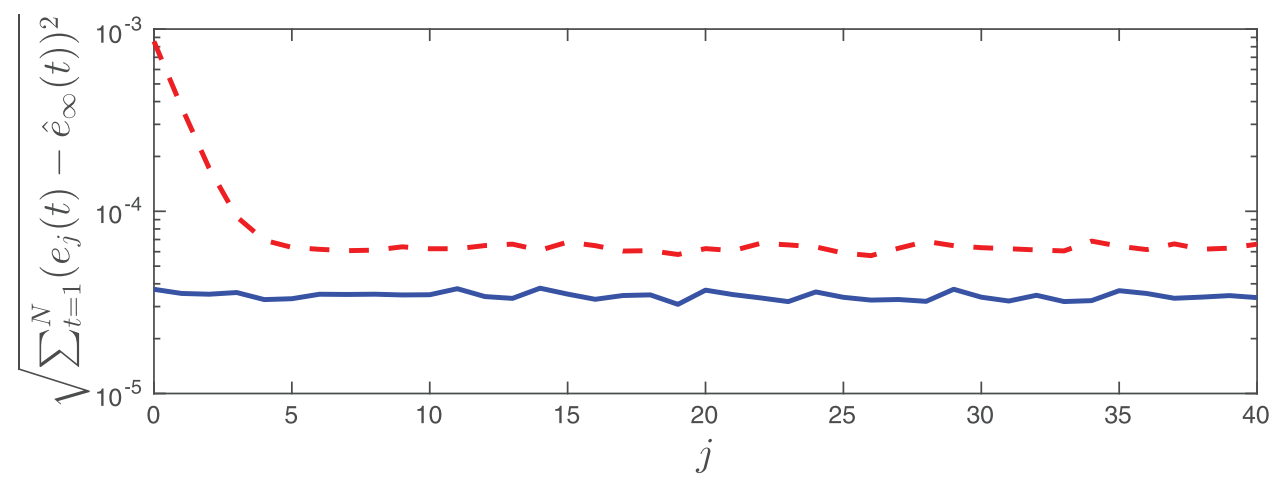

Fig. 7. Trial-varying part of the error signal $\sqrt{\sum_{t=1}^{N}\left(e_{j}(t)-\hat{e}_{\infty}(t)\right)^{2}}$ for feedback only, i.e., $f_{j}=0, j=0,1,2, \ldots$ (solid blue) and traditional norm-optimal ILC of Section 5.2 (dashed red). (For interpretation of the references to colour in this figure legend, the reader is referred to the web version of this article.)

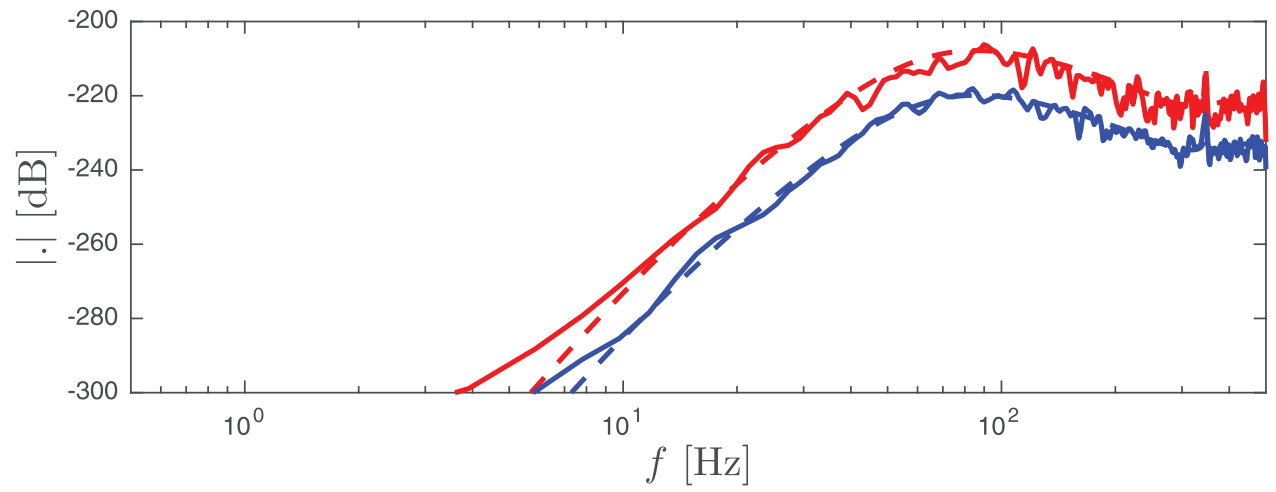

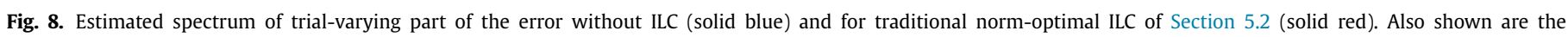

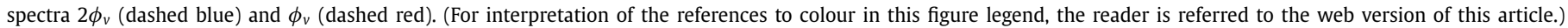




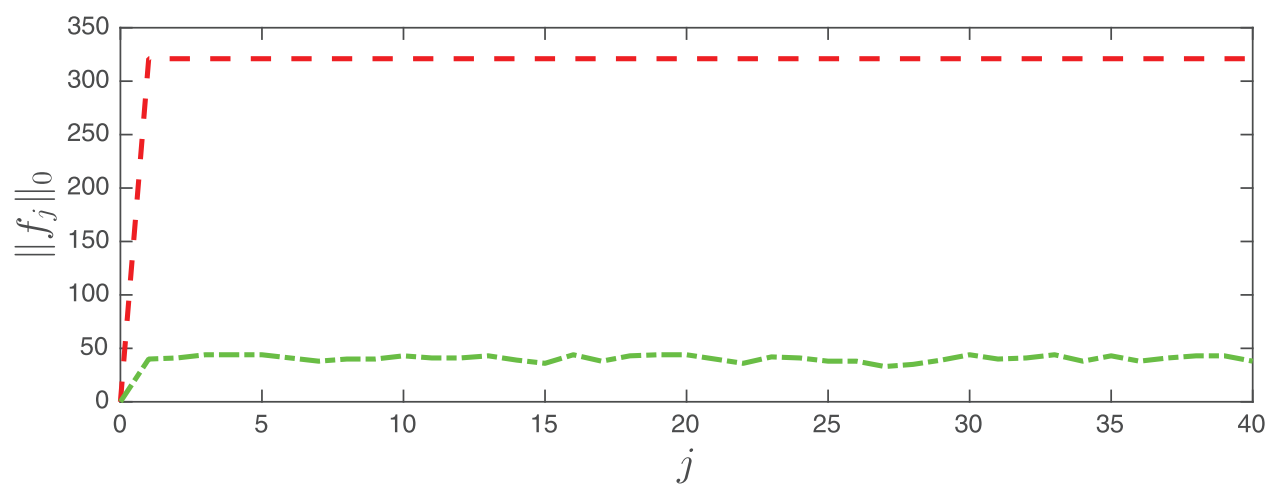

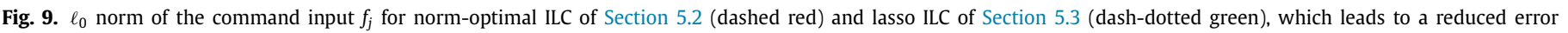
signal. (For interpretation of the references to colour in this figure legend, the reader is referred to the web version of this article.)

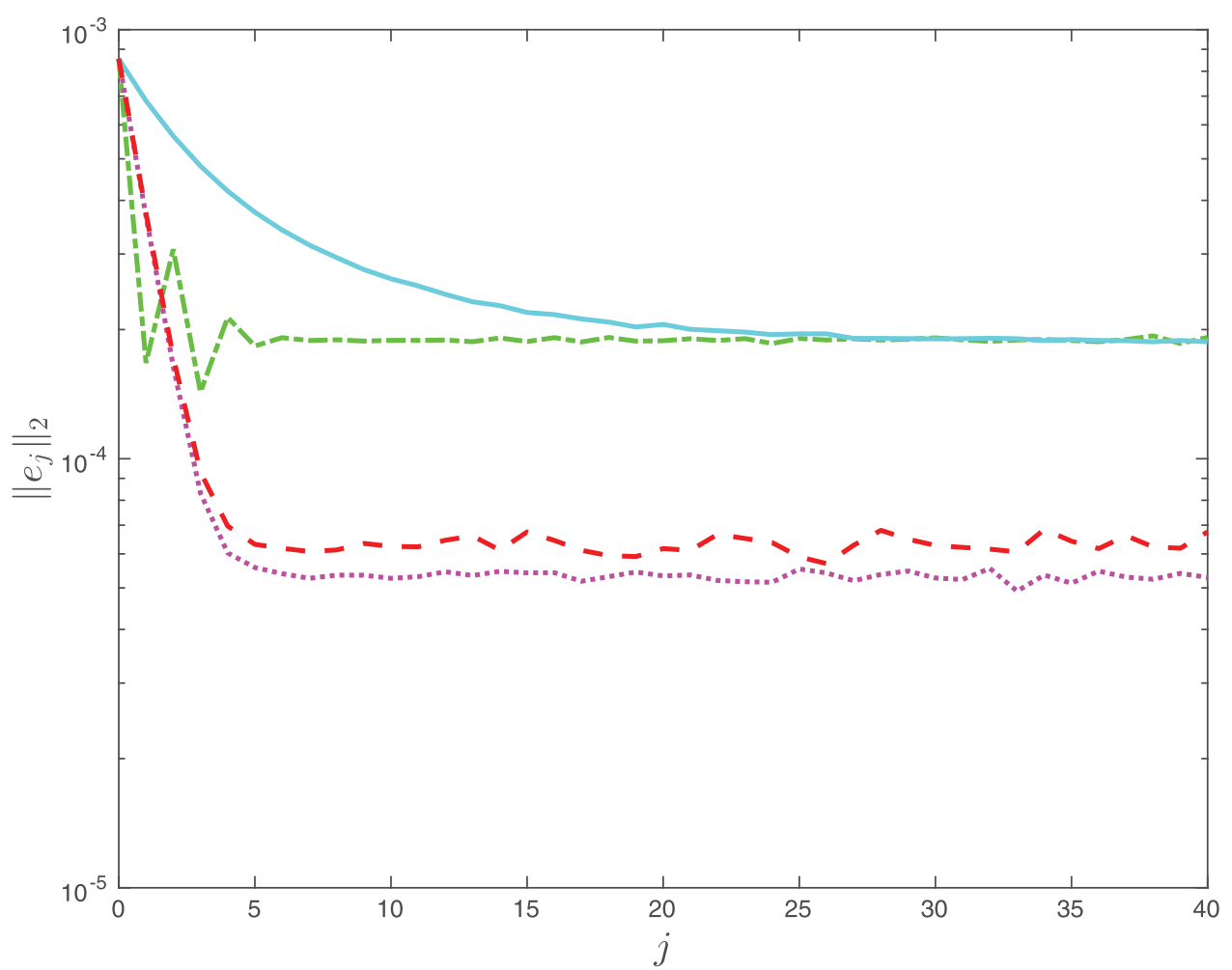

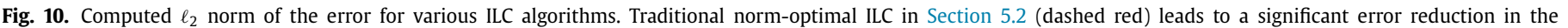

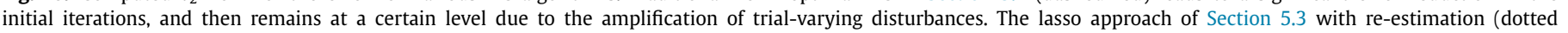

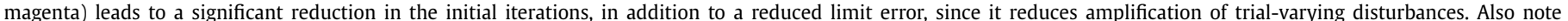

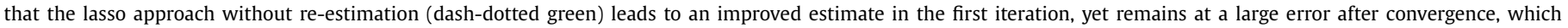

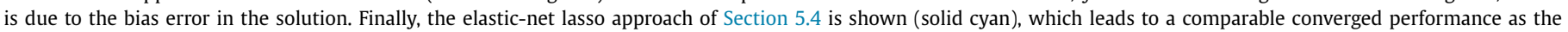

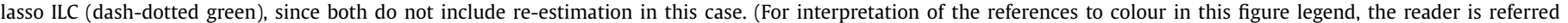
to the web version of this article.)

cluded that for non-iterative approaches, the biased estimate can be useful in terms of a bias/variance trade-off, but in the iterative schemes the benefit of re-estimation is clearly confirmed in Fig. 11.

\subsection{Elastic net lasso ILC}

In this section, the approach of Section 4.3 is pursued, where the lasso regularization is extended with a ridge regression term. In particular, $W_{f}=0$, while $W_{\Delta f}=1 \cdot 10^{-6} I$. The resulting error $e_{40}$ and command input $f_{40}$ are depicted in Fig. 12. The error is of comparable magnitude as the lasso ILC in Section 11, while the command input is substantially smoother. The error in fact has slightly reduced compared to lasso ILC, as is shown in Fig. 10, which comes at the price of a slower convergence rate due to an increased
$W_{\Delta f}$. Notice that the elastic net lasso can also be improved by reestimation, which is not done here to facilitate the presentation.

\subsection{Fused lasso ILC}

The results in the previous sections have addressed Requirement R3a. In certain situations, e.g., wireless sensors or embedded implementations, it may be required to minimize the number of times the command input is updated, i.e., Requirement R3b. This is a different form of structure compared to sparsity. To address this, the fused lasso of Section 4.4 is employed.

In particular, in the general criterion (8) is considered, where the weighting filters are selected as $W_{f}=W_{\Delta f}=0, D=D_{f}$ in (17), and $\lambda=3 \cdot 10^{-12}$. 

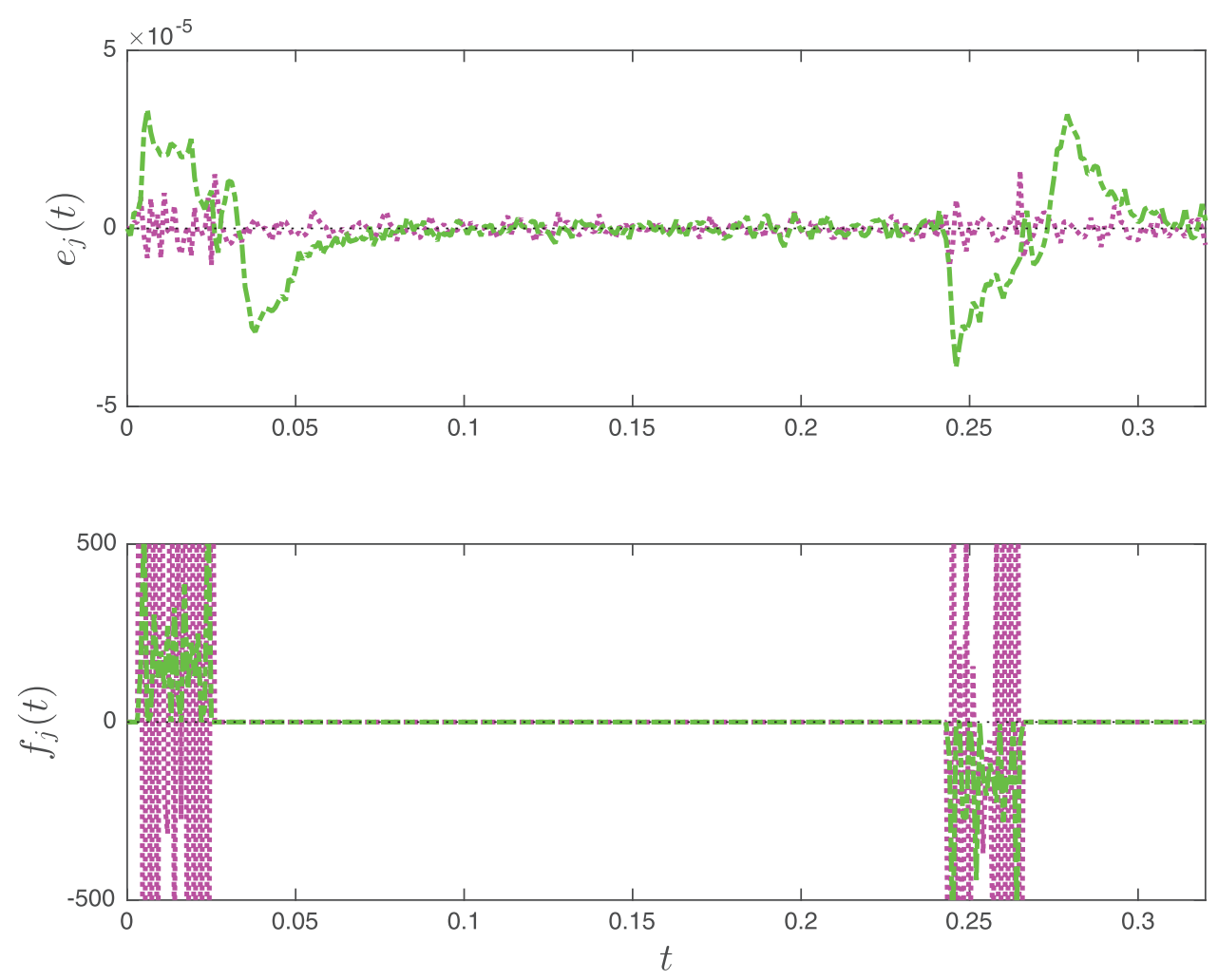

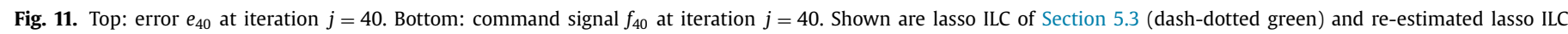
of Section 5.3 (dotted magenta). (For interpretation of the references to colour in this figure legend, the reader is referred to the web version of this article.)
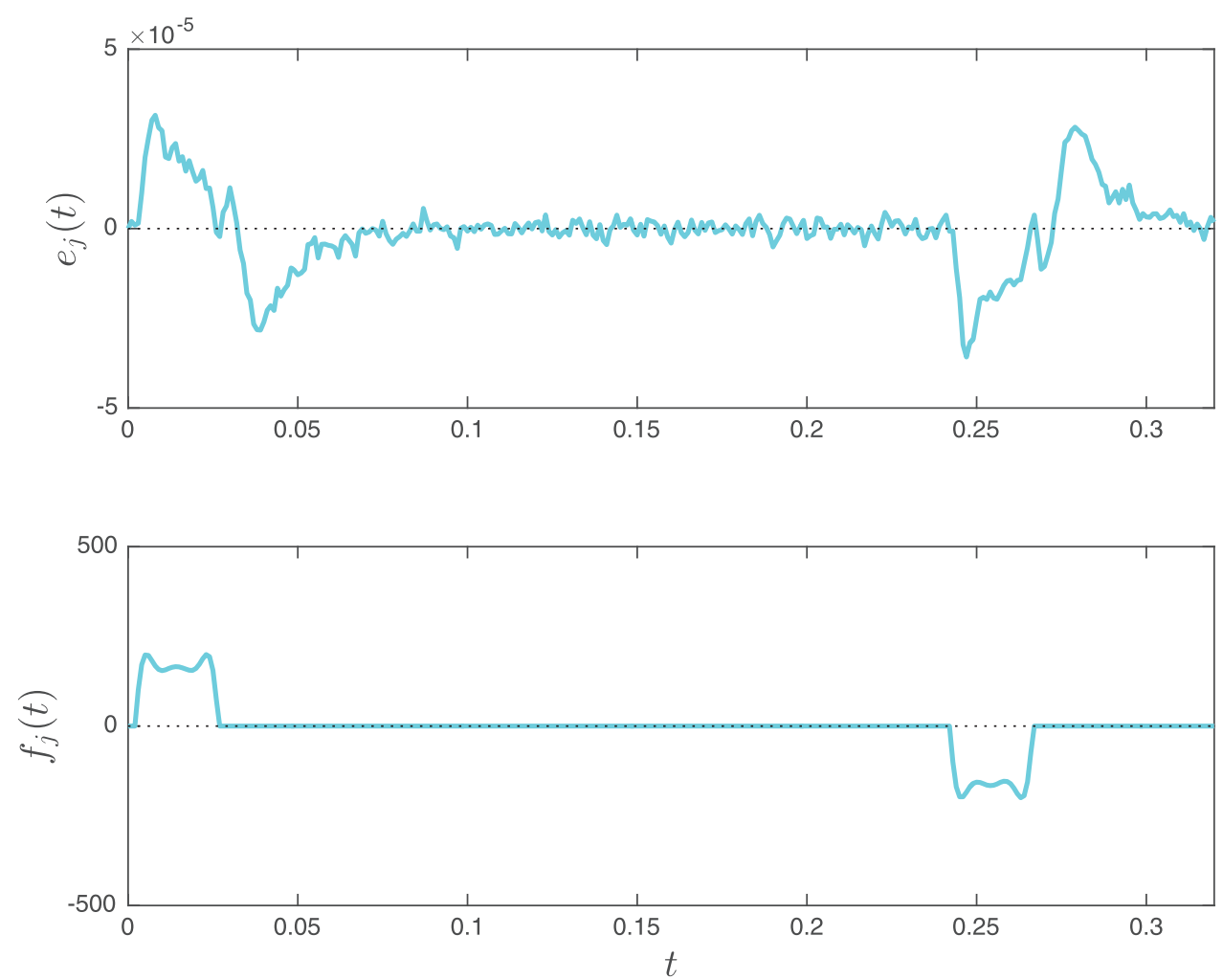

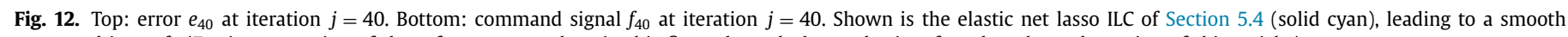
command input $f_{j}$. (For interpretation of the references to colour in this figure legend, the reader is referred to the web version of this article.) 


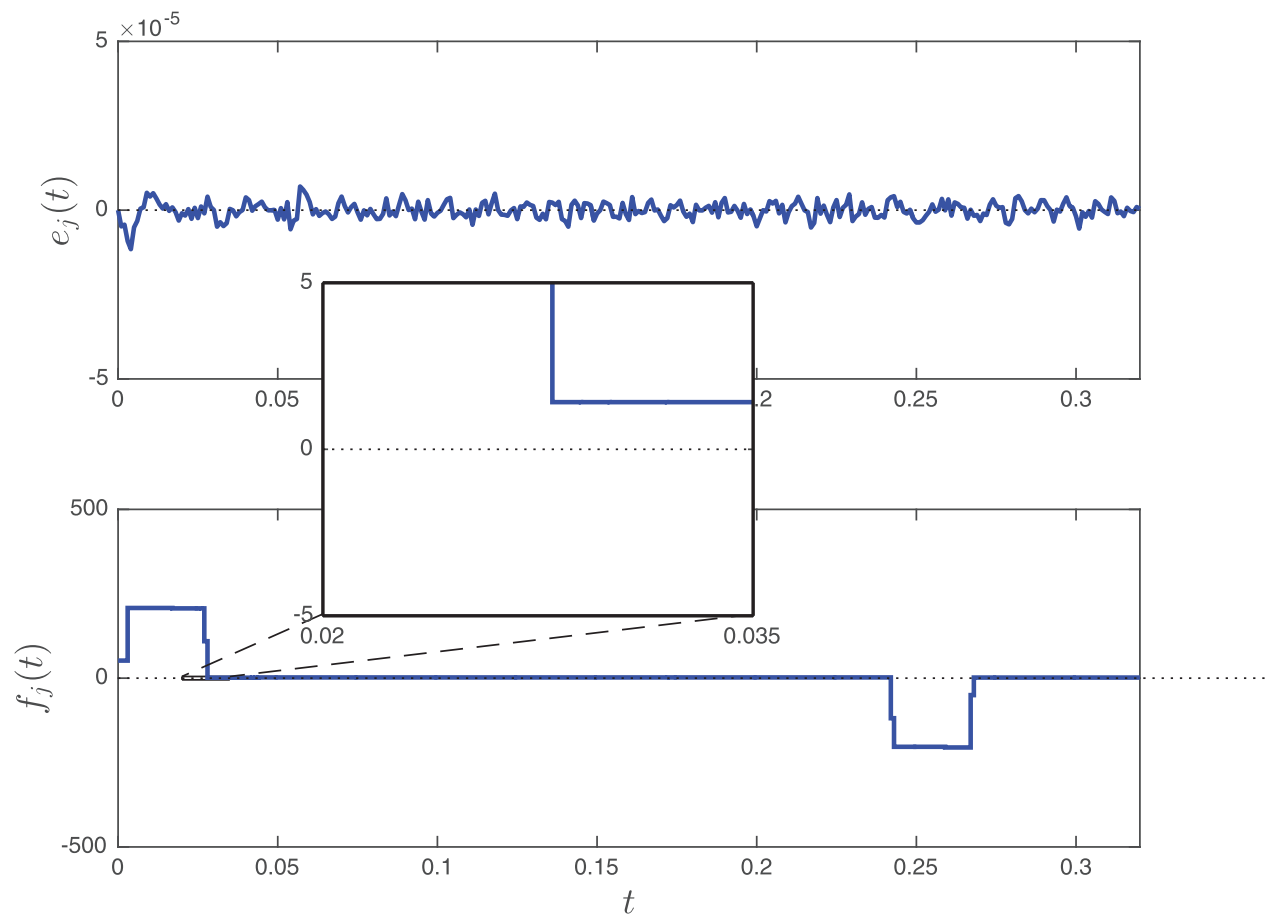

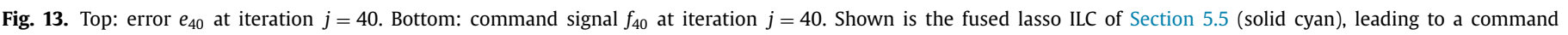

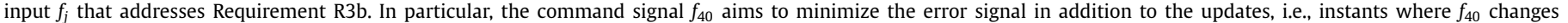

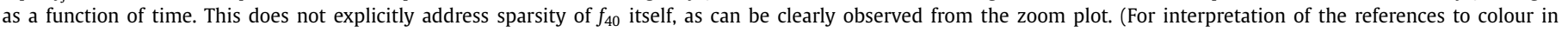
this figure legend, the reader is referred to the web version of this article.)

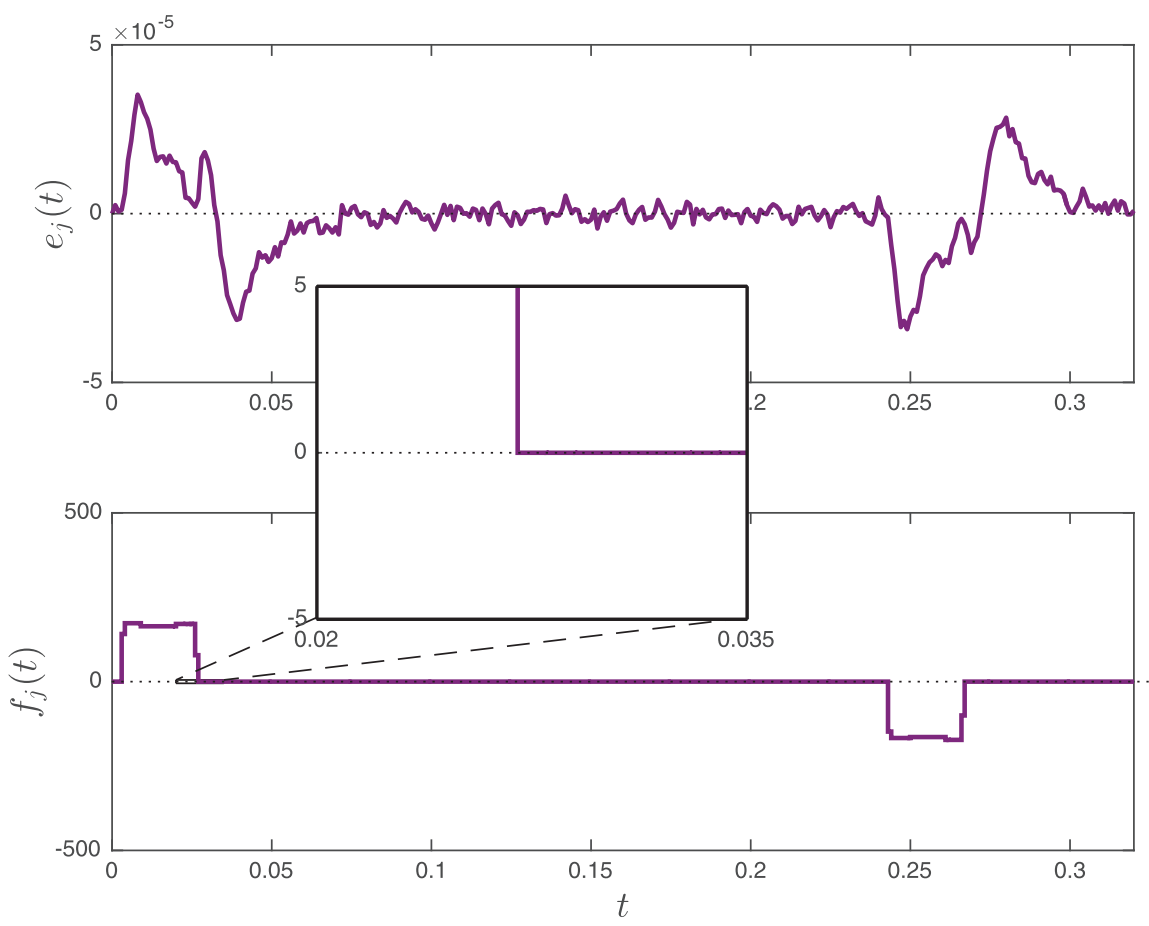

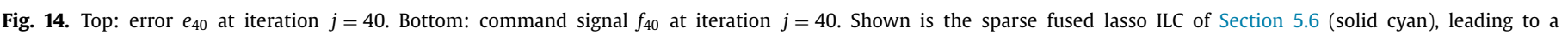

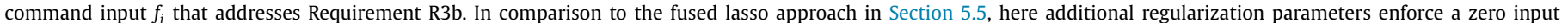

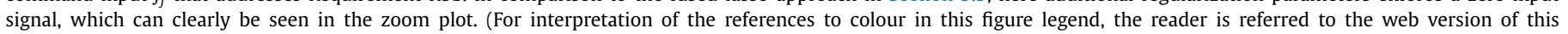
article.)

Next, the ILC iteration is invoked. The results are shown in Fig. 13. Compared to the results of Fig. 11 in Section 5.3, the error has reduced significantly. However, this comes at the price of sparsity. Indeed, only the first samples are zero, since the algorithm is initialized with $f_{1}(0)=0$. Interestingly, only a limited number of command signal updates are required to achieve a small error signal. This will also attenuate the effect of trial-varying disturbances. Note that the error can be further reduced by including a re-estimation step, which is not shown here to facilitate the presentation. 


\subsection{Sparse fused lasso ILC}

In the previous sections, Requirement R3a and Requirement R3b are achieved separately in Section 5.3 and Section 5.5, respectively. To address both requirements simultaneously, the sparse fused lasso approach of Section 4.5 is adopted. The regularization penalties in (19) are selected such that these essentially combine the two penalties in Sections 5.3 and 5.5.

The results are depicted in Fig. 14. It can directly be observed that it combines the sparsity of Section 5.3 while at the same time reducing the number of command signal updates as in Section 5.5. As such, it is concluded that the sparse fused lasso addresses Requirement R3a and Requirement R3b simultaneously. The relative penalties can be further tuned to balance the importance of both penalties, as well as the resulting error signal. In addition, the resulting error signal can be further enhanced through a reestimation step.

\section{Conclusion}

A general framework is presented that extends optimizationbased iterative learning control to include additional structure, including sparsity. The approach is shown on a mechatronic system, where it is shown to have significant benefits, including (i) resource-efficiency in terms of sparse command signals, e.g., facilitating embedded controller implementations; (ii) resourceefficiency in terms of limiting the number of changes in the command signal, e.g., facilitating implementation in limited-capacity communication networks; (iii) automated basis function selection in flexible iterative learning control employing basis functions; and (iv) attenuation of trial-varying disturbances, which for the considered wafer scanner example leads to significant performance increase. Regarding the latter, a detailed analysis of trial-varying disturbances in ILC reveals that such trial-varying exogenous signals are often amplified by typical ILC algorithms. The proposed framework enables a significant reduction of this amplification, typically up to a factor of two.

The proposed framework enables many user-specific choices, and can be easily extended. For instance, for third-order or higherorder setpoints, it may be useful to impose regularization parameters of equally high polynomial orders, known as polynomial trend filtering [52, Section 2.1.2], which is a special case of the general criterion (8).

Ongoing research focuses on specialized algorithms for the considered scenarios, enabling faster computation. In addition, the correlation between variables is subject of further investigation. Finally, various aspects of monotonic convergence, which has here been analyzed in terms of the $\ell_{1}$ norm, are being investigated, including robust monotonic convergence conditions [18,55] and data-driven ILC frameworks [7,28]. Finally, connections to recent frequency-domain designs, including $[42,48]$, where convex optimization is used to design $L$ and $Q$ filters in certain frequency ranges, e.g., to attenuate trial-varying disturbances, could enable a further improvement in the design of ILC controllers.

\section{Acknowledgments}

This paper is the result of several research visits of both authors, which is supported in part of the research programme VENI with project number 13073, which is (partly) financed by the Netherlands Organisation for Scientific Research (NWO). In addition, the authors gratefully acknowledge the fruitful discussions with Jurgen van Zundert, Maurice Heemels, Dip Goswami, and Martijn Koedam for resource-efficient control, as part of the Robust Cyber-Physical Systems (RCPS) project (no. 12694).

\section{Appendix A}

In this section, a proof of Theorem 3 is provided. Several auxiliary results are presented. In particular, note that at iteration $j$, the error is a function of all previous signals affecting the loop due to the iteration-domain integrator in (4). In the following lemma, the summation of $j$ terms of the trial-invariant disturbance $r$ in (1) is eliminated.

Lemma 5. Consider the system (1) and ILC update (4) with $f_{0}=0$ and assume that iteration is stable in the sense of Theorem 2. Then,

$$
\begin{aligned}
e_{j}= & \left(1-\tilde{J} \frac{1-(\tilde{Q}(1-\tilde{L} \tilde{J}))^{j}}{1-\tilde{Q}(1-\tilde{L} \tilde{J})} \tilde{L}\right) r \\
& -v_{j}-\tilde{J} \sum_{n=0}^{j-1}\left(\tilde{Q}(1-\tilde{L} \tilde{J})^{n} \tilde{Q} \tilde{L} v_{j-n-1} .\right.
\end{aligned}
$$

Proof. Substituting (1) into (4) yields

$f_{j+1}=\tilde{Q}\left((1-\tilde{L} \tilde{)}) f_{j}+\tilde{L} r-\tilde{L} v_{j}\right)$.

Given $f_{0}=0$ and subsequent successive substitution yields $f_{1}=$ $\tilde{Q}\left(\tilde{L} r-\tilde{L} v_{0}\right), \quad f_{2}=\tilde{Q}((1-\tilde{L} \tilde{J})+1) \tilde{Q} \tilde{L} r-\tilde{Q}(1-\tilde{L} \tilde{J}) \tilde{Q} \tilde{L} v_{0}-\tilde{Q} \tilde{L} v_{1}$, and hence

$f_{j}=\sum_{i=0}^{j-1}(\tilde{Q}(1-\tilde{L} \tilde{J}))^{i} \tilde{Q} \tilde{L} r-\sum_{n=0}^{j-1}(\tilde{Q}(1-\tilde{L} \tilde{J}))^{n} \tilde{Q} \tilde{L} v_{j-1-n}$.

Next, using the geometric series

$\sum_{l=0}^{j-1} r^{l}=\frac{1-r^{j}}{1-r}$

this leads to

$f_{j}=\frac{1-(\tilde{Q}(1-\tilde{L} \tilde{J}))^{j}}{1-\tilde{Q}(1-\tilde{L} \tilde{J})} \tilde{L} r-\sum_{n=0}^{j-1}(\tilde{Q}(1-\tilde{L} \tilde{J}))^{n} \tilde{Q} \tilde{L} v_{j-1-n}$.

Finally, substitution of (25) into (1) yields the desired result (23).

The result (23) reveals that the error contains a summation over $j$ trial-varying disturbance terms $v_{j}$, whereas the influence of the trial-invariant disturbances is captured in a single term through the use of a geometric series. Although the trial-varying disturbance varies on each experiment, a closed-form expression can be obtained by exploiting Assumption 1.

Lemma 6. Let Assumption 1 hold. Then, under the assumptions of Lemma 5,

$$
\begin{aligned}
\phi_{e_{j}}= & \mid 1-\tilde{J} \frac{1-(\tilde{Q}(1-\tilde{L} \tilde{J}))^{j}}{1-\tilde{Q}\left(1-\left.\tilde{L} \tilde{L}\right|^{2} \phi_{r}\right.} \\
& +\left(1+|\tilde{J} \tilde{Q} \tilde{L}|^{2} \frac{1-|\tilde{Q}(1-\tilde{L} \tilde{J})|^{2 j}}{1-|\tilde{Q}(1-\tilde{L} \tilde{J})|^{2}}\right) \phi_{v}
\end{aligned}
$$

Proof. Taking spectra yields

$$
\begin{aligned}
\phi_{e_{j}}= & \left|1-\tilde{J} \frac{1-(\tilde{Q}(1-\tilde{L} \tilde{J}))^{j}}{1-\tilde{Q}(1-\tilde{L} \tilde{J})} \tilde{Q}\right|^{2} \phi_{r} \\
& +\left(1+|\tilde{J} \tilde{Q} \tilde{L}|^{2} \sum_{n=0}^{j-1}\left|(\tilde{Q}(1-\tilde{L} \tilde{J}))^{n}\right|^{2}\right) \phi_{v} .
\end{aligned}
$$

Next, using (24) yields the desired result (26).

The closed-form solution (26) enables a direct proof of Theorem 3.

Proof. (of Theorem 3) Taking the limit $j \rightarrow \infty$ implies that $\mid(1-$ $\tilde{L} \tilde{J}))^{j} \mid \rightarrow 0$, directly leading to the desired result (5). 


\section{References}

[1] Ahn H-S, Moore KL, Chen Y. Iterative learning control: robustness and monotonic convergence for interval systems. Communications and control engineering series. London, United Kingdom: Springer-Verlag; 2007.

[2] Annergren M, Hansson A, Wahlberg B. An ADMM algorithm for solving $\ell_{1}$ regularized MPC. In: Proc. 2012 conf. dec. contr.; 2012. p. 4486-91. Maui, Hawaii, United States.

[3] Bach F, Jenatton R, Mairal J, Obozinski G. Optimization with sparsity-inducing penalties. volume 4 of Foundations and trends in machine learning; 2011.

[4] Bertsimas D, King A, Mazumder R. Best subset selection via a modern optimization lens. Ann Stat 2016;44(2).

[5] Boeren F, Bareja A, Kok T, Oomen T. Frequency-domain ILC approach for repeating and varying tasks: with application to semiconductor bonding equipment. IEEE Trans Mech 2016;21(6):2716-27.

[6] Boeren F, Bruijnen D, Oomen T. Enhancing feedforward controller tuning via instrumental variables: with application to nanopositioning. Int J Contr 2017;90(4):746-64.

[7] Bolder J., Kleinendorst S., Oomen T.. Data-driven multivariable ILC: enhanced performance by eliminating $L$ and $Q$ filters. Int $\mathrm{J}$ Rob Nonlin Contr, To appear. doi:10.1002/rnc.3611.

[8] Bolder J, Oomen T, Koekebakker S, Steinbuch M. Using iterative learning control with basis functions to compensate medium deformation in a wide-format inkjet printer. Mechatronics 2014;24(8):944-53.

[9] Bolder J, van Zundert J, Koekebakker S, Oomen T. Enhancing flatbed printer accuracy and throughput: optimal rational feedforward controller tuning via iterative learning control. IEEE Trans Ind Electron 2017;64(5):4207-16.

[10] Breiman L. Better subset regression using the nonnegative garrote. Technometrics 1995;37(4):373-84

[11] Bristow DA. Weighting matrix design for robust monotonic convergence in norm optimal iterative learning control. In: Proc 2008 Americ contr conf. Seattle, Washington, United States; 2008. p. 4554-60.

[12] Bristow DA, Tharayil M, Alleyne AG. A survey of iterative learning control: a learning-based method for high-performance tracking control. IEEE Control Syst Mag 2006;26(3):96-114.

[13] Bühlmann P, van de Geer S. Statistics for high-dimensional data. Springer series in statistics. Heidelberg, Germany: Springer; 2011.

[14] Butcher M, Karimi A. Advances in the theory of control, signals and systems with physical modeling. In: Iterative Learning Control Using Stochastic Approximation Theory with Application to a Mechatronic System. Springer; 2011. p. 49-64. Number 407 in LNCIS.

[15] Butler H. Position control in lithographic equipment an enabler for current-day chip manufacturing. IEEE Control Syst Mag 2011;31(5):28-47.

[16] Candès E, Wakin MB, Boyd SP. Enhancing sparsity by reweighted $\ell_{1}$ minimization. J Fourier Anal Appl 2008;14:877-905.

[17] Candès EJ, Tao T. Decoding by linear programming. IEEE Trans Inf Theory 2005:51(12):4203-15

[18] Son TD, Pipeleers G, Swevers J. Robust monotonic convergent iterative learning control. IEEE Trans Autom Control 2016;61(4):1063-8.

[19] Felici F, Oomen T. Enhancing current density profile control in tokamak experiments using iterative learning control. In: Proc. 54th conf. Dec. contr.. Japan: Osaka; 2015. p. 5370-7.

[20] Freeman C, Hughes A-M, Burridge J, Chappell P, Lewin P, Rogers E. Iterative learning control of FES applied to the upper extremity for rehabilitation. Control Eng Pract 2009;17(3):368-81.

[21] Gallieri M. $\ell_{\text {asso }}-\mathrm{MPC}$ - predictive control with $\ell_{1}$-regularised least squares. Springer theses. Switzerland: Springer; 2015.

[22] Goossens K, Azevedo A, Chandrasekar K, Gomony MD, Goossens S, Koedam M, et al. Virtual execution platforms for mixed-time-criticality systems: the compSOC architecture and design flow. SIGBED Rev 2014;10(3):23-34.

[23] Gunnarsson S, Norrlöf M. On the design of ILC algorithms using optimization. Automatica 2001:37:2011-16.

[24] Gunnarsson S, Norrlöf M. On the disturbance properties of high order iterative learning control algorithms. Automatica 2006;42:2031-4.

[25] Hastie T, Tibshirani R, Wainwright M. Statistical learning with sparsity: the Lasso and generalizations. CRC Press; 2015.

[26] Hoelzle DJ, Alleyne AG, Johnson AJW. Basis task approach to iterative learning control with applications to micro-robotic deposition. IEEE Trans Control Syst Technol 2011;19(5):1138-48.

[27] Hoelzle DJ, Barton KL. On spatial iterative learning control via 2-D convolution: stability analysis and computational efficiency. IEEE Trans Control Syst Technol 2016;24(4):1504-12.

[28] Janssens P, Pipeleers G, Swevers J. A data-driven constrained norm-optimal iterative learning control framework for LTI systems. IEEE Trans Control Syst Technol 2013;21(2):546-51.
[29] Pakazad SK, Ohlsson H, Ljung L. Sparse control using sum-of-norms regularized model predictive control. In: Proc. 2013 conf. Dec. contr. Italy: Firenze; 2013. p. 5758-63.

[30] Kim S-J, Koh W, Boyd S, Gorinevsky D. $\ell_{1}$ trend filtering. SIAM Rev 2009;51(2):339-60.

[31] Lambrechts $P$, Boerlage M, Steinbuch M. Trajectory planning and feedforward design for electromechanical motion systems. Control Eng Pract 2005; $13: 145-57$

[32] Ljung L. System identification: theory for the user. Prentice Hall, Upper Saddle River, NJ USA. 2nd; 1999.

[33] Merry R, van de Molengraft R, Steinbuch M. Iterative learning control with wavelet filtering. Int J Robust Nonlinear Control 2008;18(10):1052-71.

[34] van der Meulen S, Tousain RL, Bosgra OH. Fixed structure feedforward controller design exploiting iterative trials: application to a wafer stage and a desktop printer. J Dyn Syst Meas Control 2008;130. 051006-1

[35] Moore KL. Iterative learning control for deterministic systems. London, United Kingdom: Springer-Verlag; 1993.

[36] Murphy KP. Machine learning: a probabilistic perspective. The MIT Press; 2012

37] Natarajan BK. Sparse approximate solutions to linear systems. SIAM J Comput $1995 ; 24(2): 227-34$

[38] Ohlsson H, Ljung L, Boyd S. Segmentation of ARX-models using sum-of-norms regularization. Automatica 2010;46(6):1107-11.

[39] Oomen T. Controlling aliased dynamics in motion systems? An identification for sampled-data control approach. Int J Control 2014;87(7):1406-22.

[40] Oomen T, van Herpen R, Quist S, van de Wal M, Bosgra O, Steinbuch M. Connecting system identification and robust control for next-generation motion control of a wafer stage. IEEE Trans Control Syst Technol 2014;22(1):102-18.

[41] Owens DH. Iterative learning control: an optimization paradigm. Advances in industrial control. Springer; 2016.

[42] Paszke W, Rogers E, Gałkowski K. Experimentally verified generalized KYP lemma based iterative learning control design. Control Eng Pract 2016;53:57-67.

[43] Peng C, Sun L, Zhang W, Tomizuka M. Optimization-based constrained iterative learning control with application to building temperature control systems. In: Proc. 2016 conf. AIM. Canada: Banff, Alberta; 2016. p. 709-15.

[44] Pipeleers G, Moore KL. Unified analysis of iterative learning and repetitive controllers in trial domain. IEEE Trans Autom Contr 2014;59(4):953-65.

[45] Rogers E., Galkowski K., Owens D.H.. Control systems theory and applications for linear repetitive processes. In: Number 349 in LNCIS. Berlin, Germany: Springer; 2007.

[46] Rojas CR, Hjalmarsson H. Sparse estimation based on a validation criterion. In: Proc. 2011 conf. Dec. contr. Orlando, Florida, United States; 2011. p. 2825-30.

[47] Rojas CR, Tóth R, Hjalmarsson H. Sparse estimation of polynomial and rational dynamical models. IEEE Trans Autom Control 2014;59(11):2962-77.

[48] Son TD, Pipeleers G, Swevers J. Multi-objective iterative learning control using convex optimization. Eur J Control 2017;33:35-42.

[49] Stein C. Inadmissibility of the usual estimator for the mean of a multivariate normal distribution. In: Proceedings of the third Berkeley symposium on mathematical statistics and probability, volume 1. Univ. of Calif. Pres; 1956. p. 197-206.

[50] Tibshirani R. Regression shrinkage and selection via the lasso. J R Stat Soc 1996:58(1):267-88.

[51] Tibshirani R, Saunders M, Rosset S, Zhu J, Knight K. Sparsity and smoothness via the fused lasso. J R Stat Soc B 2005;67(1):91-108.

[52] Tibshirani RJ, Taylor J. The solution path of the generalized lasso. Ann Stat 2011:39(3):1335-71.

[53] Axehill JW, Dressler I, Gunnarsson S, Robertsson A. Estimation-based ILC applied to a parallel kinematic robot. Control Eng Pract 2014;33:1-9.

[54] van de Wijdeven J, Bosgra O. Using basis functions in iterative learning control: analysis and design theory. Int J Control 2010;83(4):661-75.

[55] van de Wijdeven J, Donkers T, Bosgra O. Iterative learning control for uncertain systems: robust monotonic convergence analysis. Automatica 2009;45:2383-91.

[56] Yuan M, Lin Y. Model selection and estimation in regression with grouped variables. J R Stat Soc Ser B 2006;68:49-67.

[57] Zhou K, Doyle JC, Glover K. Robust and optimal control. Prentice Hall, Upper Saddle River, NJ; 1996

[58] Zou H. The adaptive lasso and its oracle properties. J Am Stat Assoc 2006;101(476):1418-29.

[59] Zou H, Hastie T. Regularization and variable selection via the elastic net. J R Stat Soc B 2005;67(2):301-20

[60] van Zundert J, Bolder J, Koekebakker S, Oomen T. Resource-efficient ILC for LTI/LTV systems through LQ tracking and stable inversion: enabling large tasks on a position-dependent industrial printer. Mechatronics 2016;38:76-90.

[61] van Zundert J, Bolder J, Oomen T. Optimality and flexibility in iterative learning control for varying tasks. Automatica 2016:67:295-302. 


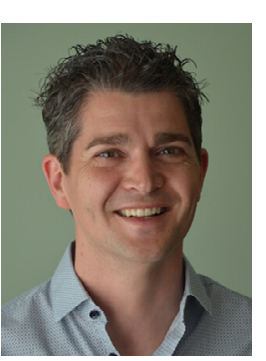

Tom Oomen received the M.Sc. degree (cum laude) and Ph.D. degree from the Eindhoven University of Technology, Eindhoven, The Netherlands. He held visiting positions at KTH, Stockholm, Sweden, and at The University of Newcastle, Australia. Presently, he is an assistant professor with the Department of Mechanical Engineering at the Eindhoven University of Technology. He is a recipient of the Corus Young Talent Graduation Award, the 2015 IEEE Transactions on Control Systems Technology Outstanding Paper Award, and the 2017 IFAC Mechatronics Best Paper Award. He is Associate Editor on the IEEE Conference Editorial Board, IFAC Mechatronics, and the IEEE Control Systems Letters (L-CSS). His research interests are in the field of system identification, robust control, and learning control, with applications in mechatronic systems.

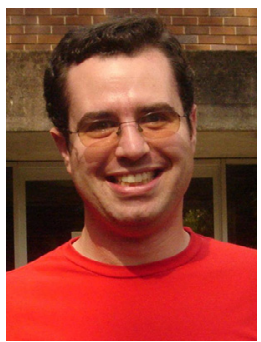

Cristian R. Rojas was born in 1980 . He received the M.S. degree in electronics engineering from the Universidad Técnica Federico Santa María, Valparaíso, Chile, in 2004, and the Ph.D. degree in electrical engineering at The University of Newcastle, NSW, Australia, in 2008. Since October 2008, he has been with the Royal Institute of Technology, Stockholm, Sweden, where he is currently Associate Professor of the Department of Automatic Control, at the School of Electrical Engineering. Dr. Rojas is a member of the IEEE Technical Committee on System Identification and Adaptive Control and the IFAC Technical Committee TC1.1. on Modelling, Identification, and Signal Processing. He is Associate Editor of Automatica and the IEEE Control Systems Letters. His research interests lie in system identification and signal processing. 